\title{
Detection of abundant bacteria in the Weser estuary using culture-dependent and culture-independent approaches
}

\author{
Natascha Selje ${ }^{1,2}$, Thorsten Brinkhoff ${ }^{1}{ }^{,}$Meinhard Simon ${ }^{1, *}$ \\ ${ }^{1}$ Institute for Chemistry and Biology of the Marine Environment, University of Oldenburg, 26111 Oldenburg, Germany \\ ${ }^{2}$ Present address: Institut für Tierproduktion in Tropen und Subtropen, Universität Hohenheim, Fruwirthstraße 12, \\ 70599 Stuttgart, Germany
}

\begin{abstract}
To assess the composition and abundant members of the bacterial community in the Weser estuary, Germany, we applied a PCR-dependent approach simultaneously with a PCRindependent approach, the dilution culture technique. Dilution cultures growing in autoclaved ambient water without any nutrient addition from the limnetic, brackish and marine sections of the Weser estuary from May, August and November 1999 yielded growth to a dilution of $10^{-6}$ to $10^{-9}$, equivalent to a cultivation efficiency of 1.5 to $66 \%$. Bacteria enriched in the highest dilution steps were analyzed by denaturing gradient gel electrophoresis (DGGE) of PCR-amplified 16S rRNA gene fragments. The results show that with increasing dilution steps, fewer (in 7 cases only 1) DGGE bands occurred that matched prominent bands in the DGGE patterns of the natural bacterial community. In the 7 cases with only 1 band, almost complete 16S rRNA gene sequences were retrieved, thus extending the limited sequence information provided by the DGGE approach. The dominant phylotypes included bacteria of clusters within $\alpha$ - and $\beta$-Proteobacteria, the Flavobacteria/Sphingobacteria group of the Bacteroidetes phylum and Actinobacteria, comprising cultivated strains but also clusters of as yet uncultured phylotypes. Closely related bacteria and phylotypes in most of these clusters are well known from other limnetic and marine systems. We also obtained isolates from the high dilution steps. More than $30 \%$ of them, belonging to various clusters within $\alpha$ - and $\beta$-Proteobacteria and the Flavobacteria/Sphingobacteria group, had a sequence similarity of $<96 \%$ to described species. $\alpha$-Proteobacteria constituted the most abundant phylotypes of the DGGE bands in the high dilution steps and of isolates, whereas Flavobacteria/Sphingobacteria constituted only low proportions. There were pronounced differences in the composition of bacterial communities on solid- and liquid-phase media in enrichment cultures with algal extracts and a preferential growth of $\gamma$-Proteobacteria that did not occur in the natural samples and the high dilution steps.
\end{abstract}

KEY WORDS: Estuaries · Bacteria $\cdot$ DGGE $\cdot$ Dilution culture $\cdot 16 \mathrm{~S}$ rRNA gene sequences

\section{INTRODUCTION}

Aquatic microbial ecology has long been challenged by the fact that only a minor fraction, i.e. much less than $1 \%$, of the natural bacterioplankton community enumerated by light or epifluorescence microscopy can be cultivated, preferentially on solid media (Razumov 1932, Jannasch 1958, Kuznetsov 1975, Staley \& Konopka 1985, Eguchi \& Ichida 1990). During the 1970s and 1980s, increasing evidence accumulated that the major fraction of the as yet uncultured bacteria actively grows and participates in the flux of energy and cycling of nutrients in aquatic ecosystems (van Es \& Meyer-Reil 1982, Cole et al. 1988). The development and application of molecular techniques based on PCR, such as the establishment of clone libraries and denaturing gradient gel electrophoresis (DGGE) of 16S rRNA gene sequences, revealed that the bacterio- 
plankton community is constituted of many bacteria and phylotypes that had not been detected by culturebased techniques (Giovannoni et al. 1990, Schmidt et al. 1991, Fuhrman et al. 1993, Muyzer et al. 1993, Zwart et al. 1998, Giovannoni \& Rappé 2000, Urbach et al. 2001). In fact, the application of fluorescence in situ hybridization (FISH) of rRNA-targeted oligonucleotides indicated that readily culturable bacteria account for only a minor fraction of the natural bacterial community (Eilers et al. 2000b, 2001).

The molecular approaches, even though they have opened a new realm of aquatic microbial ecology, nonetheless have shortcomings. Phylogenetic identification on the basis of the 16S rRNA gene sequence provides little information about the physiological properties of the respective phylotypes unless primers target specific physiological groups such as Cyanobacteria or ammonium oxidizers within $\beta$-Proteobacteria (Nübel et al. 1997, Ward et al. 1997, Phillips et al. 1999). Clone libraries yield almost complete $16 \mathrm{~S}$ rRNA gene sequences, but are not suitable for quantifying the abundance of the clones. DGGE detects phylotypes, which account for more than $1 \%$ of the total bacterial community, but is usually applied to amplify fragments of only 250 to $500 \mathrm{bp}$ of the 16S rRNA gene, and thus provides limited information for phylogenetic analyses (Muyzer et al. 1993, Murray et al. 1996). Further, it has been shown that particular phylotypes or phylogenetic groups are selectively amplified by PCR from mixed templates (Reysenbach et al. 1992, Farelli et al. 1995, Suzuki \& Giovannoni 1996, Gonzalez \& Moran 1997, Polz \& Cavanaugh 1998, Ishii \& Fukui 2001). Comparing clone libraries and FISH, evidence revealed that PCR amplification is not equally efficient for $\alpha$-Proteobacteria and the Cytophaga/Flavobacteria group (Cottrell \& Kirchman 2000), equivalent to the renamed Flavobacteria/Sphingobacteria group of the Bacteroidetes phylum. Hence, there is a need for characterizing and quantifying the dominant members of the natural bacterioplankton community in aquatic systems and for examining the validity of the results obtained by these techniques, preferentially by PCRindependent approaches.

One promising approach to enrich and isolate members of the bacterioplankton community is the dilution culture technique, involving the addition of nutrients at low concentrations, signal molecules, or no additional nutrients (Button et al. 1993, Bussmann et al. 2001, Jaspers et al. 2001, Bruns et al. 2002, 2003a,b, Rappé et al. 2002). It allows for growth of the most abundant bacteria in high dilution steps without the competition of less abundant but possibly faster-growing bacteria. The application of this technique led to the cultivation of new bacterial isolates from various ecosystems and increased the cultivation efficiency by up to $20 \%$.
The aim of our study was to analyze the composition of the bacterial communities in the salinity gradient of the Weser estuary, Germany, using a PCR-based approach (DGGE) simultaneously with the most promising culture dependent approach, the dilutionto-extinction technique and subsequent isolation. In a previous paper we reported on the composition and seasonal dynamics of the ambient bacterial community on the basis of DGGE (Selje \& Simon 2003). Here, we present comparisons between sequences of the most prominent bands of the DGGE patterns of natural samples with sequences retrieved from the highest dilution steps and isolates. The results show that with increasing dilution steps fewer and eventually only 1 single band occurred which matched prominent bands in the DGGE patterns of the natural bacterial community. These obviously dominant phylotypes included clusters of bacteria comprising cultivated strains but also clusters of yet uncultured phylotypes. Subsequent enrichment and isolation revealed pronounced differences with respect to growth on liquid and solid media with and without substrate additions.

\section{MATERIALS AND METHODS}

Study area and sampling. Samples were collected in the salinity gradient of the Weser estuary, Germany, in May, August and November 1999 on board the RV 'Bakensand' from $1 \mathrm{~m}$ depth by a low-pressure pump during the outflowing tide at $\mathrm{Km} 80$ (marine section), the turbidity maximum (TM, brackish section) and at $\mathrm{Km} 0$ (limnetic section). Samples were kept at $4^{\circ} \mathrm{C}$, transferred to the laboratory within $8 \mathrm{~h}$, and processed further immediately. Further details on the Weser estuary are given in Selje \& Simon (2003).

Dilution cultures. We prepared dilution series in triplicates with 1:10 steps to a dilution of $10^{-10}$. To minimize changes in growth conditions, natural unfiltered water samples from the sampling sites in the limnetic, brackish and marine sections were used as growth media. Instead of $0.2 \mu \mathrm{m}$ filtration, autoclaving was used for sterilization. We are aware that this treatment may raise the concentration of labile substrates due to dissolution and hydrolysis of particulate organic matter and polymers, but we intended to include particulates as substrate sources; filtration through $0.2 \mu \mathrm{m}$ pore filters may also allow ultramicrobacteria to pass (Haller et al. 2000, Hahn et al. 2003). We then added $1 \mathrm{ml}$ of a $0.2 \mu \mathrm{m}$ filtered trace element solution $(2.1 \mathrm{~g}$ $\mathrm{FeSO}_{4} \times 7 \mathrm{H}_{2} \mathrm{O} ; 5.2 \mathrm{~g} \mathrm{Na}_{2} \mathrm{EDTA}_{4}, 30 \mathrm{mg} \mathrm{H}_{3} \mathrm{BO}_{3}, 100 \mathrm{mg}$ $\mathrm{MnCl}_{2} \times 4 \mathrm{H}_{2} \mathrm{O}, 190 \mathrm{mg} \mathrm{CoCl} 2 \times 6 \mathrm{H}_{2} \mathrm{O}, 24 \mathrm{mg} \mathrm{NiCl}{ }_{2} \times$ $6 \mathrm{H}_{2} \mathrm{O}, 144 \mathrm{mg} \mathrm{ZnSo}_{4} \times 7 \mathrm{H}_{2} \mathrm{O}, 36 \mathrm{mg} \mathrm{Na}_{2} \mathrm{MoO}_{4} \times$ $2 \mathrm{H}_{2} \mathrm{O}, 1000 \mathrm{ml}$ aqua dest) and $2 \mathrm{ml}$ of a $0.2 \mu \mathrm{m}$ filtered vitamin solution (Schut et al. 1993) $1000 \mathrm{ml}^{-1}$ medium. 
All culture tubes and media flasks were combusted at $500^{\circ} \mathrm{C}$ for $2 \mathrm{~h}$ prior to use. The water samples were well-mixed before inoculation. Tubes containing $9 \mathrm{ml}$ of media were inoculated with $1 \mathrm{ml}$ of sample water of the respective section of the estuary, vigorously mixed, and incubated in the dark on a shaker (100 rpm) at in situ temperature $\left(15^{\circ} \mathrm{C}\right.$ in May, $20^{\circ} \mathrm{C}$ in August, $11^{\circ} \mathrm{C}$ in November). Cultures were incubated for 10 to $12 \mathrm{wk}$, and $1 \mathrm{ml}$ was then transferred to new tubes with $9 \mathrm{ml}$ of the respective media. The remaining samples were harvested by centrifugation for $15 \mathrm{~min}$ at $132.6 \times g$ (relative centrifugal force) and kept at $-20^{\circ} \mathrm{C}$ until further processing. Growth of bacteria in the dilution tubes was determined by epifluorescence microscopy and DGGE analysis. The number of cultivable bacteria (+95\% confidence intervals) was calculated by the most probable number (MPN) program of Klee (1993).

Bacteria cell counts. Total bacterial numbers were determined on black $0.2 \mu \mathrm{m}$ Nuclepore filters by epifluorescence microscopy after staining with DAPI $(4,6-$ diamidinophenylindole). For further details see Selje \& Simon (2003).

Molecular analysis. Nucleic acids were extracted by a standard protocol with phenol/chloroform slightly modified as described previously (Selje \& Simon 2003). Fragments of bacterial 16S rRNA genes were amplified with primers GM5f and 907rm (Muyzer et al. 1998) and a touchdown program with decreasing annealing temperatures from 65 to $55^{\circ} \mathrm{C}$ ( 2 cycles per step plus additional 16 cycles) with a total of 38 cycles; $1 \mu l$ (1 to $10 \mathrm{ng}$ ) of extracted DNA served as the template. DGGE analysis of the PCR products was performed according to Muyzer et al. (1998), and the cluster analysis of DGGE profiles used Pearson's correlation and UPGMA. (For further details see Selje \& Simon 2003.)

Isolation of bacteria. Aliquots (50 to $100 \mu \mathrm{l}$ ) from the higher dilution steps $\left(10^{-4}\right.$ to $\left.10^{-9}\right)$ were spread onto plates prepared with the media of natural samples (see above) and $0.9 \%$ agar (Scharlau Chemie). Plates were incubated at the corresponding in situ temperature in the dark until colonies became visible. Most colonies appeared within $1 \mathrm{mo}$; some, however, appeared only after 2 to $3 \mathrm{mo}$ and had diameters of only 1 to $2 \mathrm{~mm}$. Colonies for further transfer onto fresh plates were selected according to morphology and color. After the first transfer colonies grew faster and became bigger. To obtain pure cultures, colonies were transferred at least 3 times. Aliquots of isolates were harvested in $30 \%$ glycerol and stored at $-70^{\circ} \mathrm{C}$.

Bacterial growth on algal extracts and solid/liquid media. In May at the TM, we examined differences in the bacterial communities growing on solid- and in liquid-phase media and enriched with extracts of the green algae Scenedesmus sp. or a mixed diatom community. Algal cultures were harvested by centrifuga- tion and boiled for 5 min to extract the organic matter. Enrichment of the algal extracts was 5-fold that of ambient phytoplankton concentrations. The $10^{-3}$ dilution step of the dilution series in the brackish medium served as inoculum and was further diluted $10^{4}$-fold in the enriched liquid media. After $4 \mathrm{~d}$ of pre-incubation of duplicates, $100 \mu \mathrm{l}$ were spread out onto the agar plates with the algal extracts and incubated at $15^{\circ} \mathrm{C}$ in the dark for $2 \mathrm{wk}$. Liquid cultures and colonies from the agar plates were then harvested for DNA extraction and DGGE analysis.

Sequencing and phylogenetic analysis. DGGE bands and isolates were sequenced using different combinations of the primers GM3f, GM1r, GM5f, GM8r, 907rm and GM4r (Muyzer et al. 1993, 1995, 1998). Phylogenetic analysis was performed with the ARB software package (available at: www.arb-home.de) (Ludwig et al. 2004). Phylogenetic trees were calculated using maximum-likelihood analysis of almost full-length 16S rRNA gene sequences (>1300 bp). About 140 to 150 sequences from different families, as suggested in the prokaryotic representative listing in the Ribosomal Database Project II (RDP 2000; available at: http:// rdp.cme.msu.edu/html/) and sequences of closest relatives found by a BLAST (basic local alignment search tool) search were used to construct the backbone trees. A filter was constructed for every phylogenetic group analyzed. Hence, alignment positions at which less than $50 \%$ of sequences of the corresponding data set had the same residues were excluded from the calculations to prevent uncertain alignments within highly variable positions. Shorter sequences were added later by the maximum parsimony method, using the same $50 \%$ filter. Our proposal of new clusters is based on the finding of distinct groups of phylotypes and isolates in our samples. We did not apply any fixed percent similarity.

Nucleotide sequence accession numbers. The sequences obtained in this study are available from GenBank under Accession Nos. AY145529-AY145573 (isolates), AY145574-AY145629 (dilution cultures), and AY146656-AY146671 (enrichment cultures).

\section{RESULTS}

\section{Dilution cultures}

Dilution cultures from the various sections of the Weser estuary yielded growth to a dilution of $10^{-6}$ or $10^{-9}$ in at least 1 parallel. Growth at the fairly low dilution step of only $10^{-6}$ occurred in May 1999 with the marine inoculum, whereas growth at $10^{-9}$ was reached with the brackish inoculum in August 1999. Cultivation efficiency (MPN counts for total cell counts) ranged 


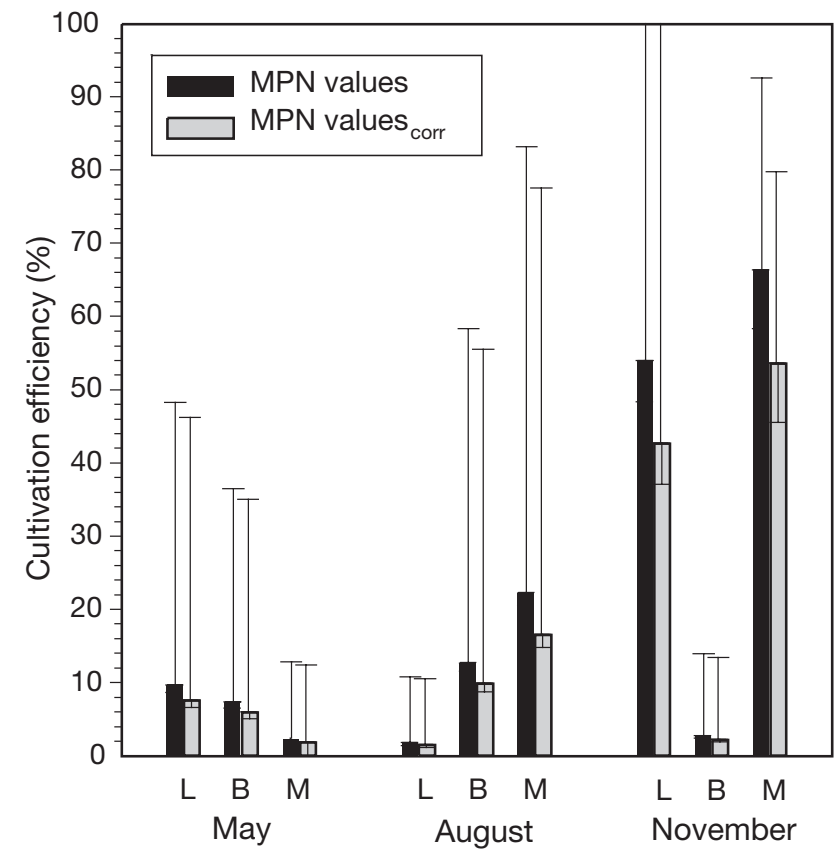

Fig. 1. Cultivation efficiencies (with 95\% confidence intervals) in limnetic (L), brackish (B) and marine (M) dilution series in May, August and November 1999 in the Weser estuary, showing most probable number (MPN) values as percent of DAPI cell counts. MPN $_{\text {corr }}$ : values corrected according to Salama (Klee 1993)

from 1.5 to $66.4 \%$, with no obvious pattern between the different sections (Fig. 1). In May, cultivation efficiency remained below $10 \%$, whereas in November it increased to $>50 \%$ in the limnetic and marine sections.
DGGE banding patterns of all dilution cultures showed a decreasing diversity with increasing dilution steps, indicating that less abundant bacteria were diluted to extinction, such as Band $\mathrm{X}$ in Fig. 2C. Some bands appeared only in the higher dilution steps, e.g. Bands DC 5-0-4, DC 5-0-6, and DC 5-80-5 (Fig. 2A-C), suggesting that the bacteria represented by these bands were outcompeted by less abundant but faster-growing species in the lower dilution steps.

In 7 cases only 1 visible band was detected by DGGE analysis in the highest dilution step with a positive growth signal. Hence, almost complete 16S rRNA gene sequences could be determined by direct amplification of the extracted DNA templates from the dilution cultures with Primer Pair GM3f and GM4r (Table 1). Among these sequences 4 phylotypes affiliated with $\alpha$-Proteobacteria: 2 almost identical sequences (>99\%, $1390 \mathrm{bp}$ ) were obtained in the marine medium at different sampling dates, and 2 sequences in the brackish medium. In the dilution cultures from November, 1 sequence from the limnetic and 1 from the brackish medium belonged to $\beta$-Proteobacteria, and 1 sequence from the marine section in August to $\gamma$-Proteobacteria. In some cases, bands from the highest dilution steps matched one of the intensive bands from the corresponding natural undiluted sample, such Bands DC 50-4 and DC 5-0-2 (Fig. 2A), and DC 5-80-2 (Fig. 2C). In total, 12 sequenced bands from the highest dilution steps of $\alpha$-Proteobacteria, $3 \beta$-Proteobacteria and 2 Actinobacteria exhibited sequence similarities of $>97 \%$ to bands from the corresponding natural undiluted sample (Table 2). Further, 10 isolates of $\alpha$-Proteobac-

Table 1. Sequences obtained from DGGE analyses of dilution cultures (DC) from the Weser estuary, showing band designation, dilution step, accession number (Acc. no.), number of base pairs, phylogenetic affiliation, closest relatives according to BLAST search, and sequence similarity. Sequence designation is: DC (dilution culture) following by month of sampling in 1999 (5 = May; 8 = August; $11=$ November) estuarine section $(0=$ limnetic $; 48 / 50 / 51=$ turbidity maximum/brackish; $80=$ marine $)$ number of excised band. Flavo: Flavobacteria/ Sphingobacteria; Uncult.; uncultured

\begin{tabular}{|c|c|c|c|c|c|}
\hline $\begin{array}{l}\text { Sequence } \\
\text { (dilution) }\end{array}$ & Acc. no. & n (bp) & Affiliation & Closest relatives (Acc. no.) & $\underset{(\%)}{\text { Similarity }}$ \\
\hline DC 5-0-1 (D7) & AY145574 & 512 & Actinobacteria & $\begin{array}{l}\text { Actinomycetales clone ACK-M1 (U85190), uncult. } \\
\text { actinobacterium WB5-41 (AF497875) }\end{array}$ & 98,94 \\
\hline DC 5-0-2 (D7) & AY145575 & 503 & Actinobacteria & $\begin{array}{l}\text { Uncult. actinobacterium WL5-10 (AF497893), Crater Lake } \\
\text { clone CL500-95 (AF316665) }\end{array}$ & 96,94 \\
\hline DC 5-0-3 (D7) & AY145576 & 512 & Actinobacteria & $\begin{array}{l}\text { Uncult. bacterium FukuN30 (AJ289996), uncult. actinobacterium } \\
\text { WL11-2 (AF497897) }\end{array}$ & 98,96 \\
\hline DC 5-0-4 (D7) & AY145577 & 508 & $\alpha$-Proteobacteria & $\begin{array}{l}\text { Uncult. } \alpha \text {-proteobacterium WL5-15 (AF497880), } \\
\text { Brevundimonas sp. (AJ227794) }\end{array}$ & 100,100 \\
\hline DC 5-0-5 (D7) & AY145578 & 506 & Flavo & Unidentified cytophagales clone LD30 (AJ007874) & 91 \\
\hline DC 5-0-6 (D6) & AY145579 & 523 & $\alpha$-Proteobacteria & $\begin{array}{l}\text { Uncult. clone HP1B64 (AF502217), 'Paracoccus kawasakiensis' } \\
\text { (AB041770) }\end{array}$ & 97,97 \\
\hline DC 5-0-7 (D6) & AY145580 & 486 & $\beta$-Proteobacteria & $\begin{array}{l}\text { Uncult. } \beta \text {-proteobacterium WL11-5 (AF497889), uncult. } \\
\beta \text {-proteobacterium ESR } 9 \text { (AF268293) }\end{array}$ & 97,97 \\
\hline DC 5-0-8 (D6) & AY145581 & 527 & $\beta$-Proteobacteria & $\begin{array}{l}\text { Uncult. } \beta \text {-proteobacterium WL5-9 (AF497885), Aquaspirillum } \\
\text { delicatum (AF078756) }\end{array}$ & 99,97 \\
\hline DC 5-50-1 (D8) & AY145582 & 1395 & $\alpha$-Proteobacteria & $\begin{array}{l}\text { Rhodobacter sp. TCRI2́ (AB017798), Rhodobacter capsulatus } \\
\text { (D16428) }\end{array}$ & 96,96 \\
\hline DC 5-50-8 (D5) & AY145583 & 504 & Flavo & Uncult. Cytophagales ESR 2 (AF268286) & 95 \\
\hline DC 5-50-11 (D5) & AY145584 & 504 & $\beta$-Proteobacteria & $\begin{array}{l}\text { Uncult. } \beta \text {-proteobacterium WL5-9 (AF497885), uncult. bacterium } \\
\text { GKS2-122 (AJ290026) }\end{array}$ & 99,98 \\
\hline DC 5-50-12 (D5) & AY145585 & 490 & $\alpha$-Proteobacteria & Sphingomonas sp. MBICC3020 (AB025279) & 97 \\
\hline DC 5-50-13 (D5) & AY145586 & 490 & $\alpha$-Proteobacteria & Sphingomonas sp. MBIC3020 (AB025279) & 97 \\
\hline
\end{tabular}


Table 1 (continued)

\begin{tabular}{|c|c|c|c|c|c|}
\hline $\begin{array}{l}\text { Sequence } \\
\text { (dilution) }\end{array}$ & Acc. no. & n (bp) & Affiliation & Closest relatives (Acc. no.) & $\underset{(\%)}{\text { Similarity }}$ \\
\hline DC 5-80-1 (D6) & AY145587 & 456 & $\alpha$-Proteobacteria & $\begin{array}{l}\text { Uncult. } \alpha \text {-proteobacterium MB12F11 (AY033316), Sinorhizobium sp. } \\
\text { DY11 (AF298836) }\end{array}$ & 97,89 \\
\hline DC 5-80-2 (D6) & AY145588 & 523 & $\alpha$-Proteobacteria & $\begin{array}{l}\text { Uncult. Roseobacter NAC11-3 (AF245632), uncult. } \alpha \text {-proteobacteria } \\
\text { WM11-37 (AF497861) }\end{array}$ & 99,99 \\
\hline DC 5-80-3 (D6) & AY145589 & 1390 & $\alpha$-Proteobacteria & Uncult. Roseobacter NAC11-3 (AF245632) & 99 \\
\hline DC 5-80-4 (D6) & AY145590 & 511 & $\alpha$-Proteobacteria & $\begin{array}{l}\text { Uncult. Roseobacter NAC11-3 (AF245632), uncult. } \alpha \text {-proteobacteria } \\
\text { WM11-37 (AF497861) }\end{array}$ & 100,100 \\
\hline DC 5-80-5 (D5) & AY145591 & 523 & $\alpha$-Proteobacteria & $\begin{array}{l}\text { Sphingomonas sp. Ant17 (AF184222), Sphingomonas sp. MBIC } 3020 \\
\text { (AB025279) }\end{array}$ & 99, 99 \\
\hline DC 5-80-6 (D5) & AY145592 & 522 & $\alpha$-Proteobacteria & Sinorhizobium xinjiangensis (AF250355) & 94 \\
\hline DC 5-80-7 (D5) & AY145593 & 523 & $\alpha$-Proteobacteria & Marine bacterium SCRIPPS_101 (AF359537) & 99 \\
\hline DC 5-80-8 (D5) & AY145594 & 537 & $\alpha$-Proteobacteria & $\begin{array}{l}\text { Marine } \alpha \text {-proteobacterium ĀS-21 (AJ391182), Roseobacter sp. } \\
\text { KT0202a (AF305498) }\end{array}$ & 100,99 \\
\hline DC 8-0-1 (D7) & AY145595 & 347 & $\alpha$-Proteobacteria & $\begin{array}{l}\text { Uncult. } \alpha \text {-proteobacterium WL11-11 (AF497882), } \alpha \text {-proteobacterium } \\
\text { F0813 (AF235997) }\end{array}$ & 99,98 \\
\hline DC 8-0-2 (D6) & AY145596 & 391 & $\alpha$-Proteobacteria & $\begin{array}{l}\text { Uncult. bacterium BUG-236 (AJ344203), Mesorhizobium sp. Lo7-12 } \\
\text { (AF410897) }\end{array}$ & 99,93 \\
\hline DC 8-0-3 (D5) & AY145597 & 360 & Flavo & $\begin{array}{l}\text { Uncult. CFB group bacterium TAF-A67 (AY038748), Flavobacterium } \\
\text { aquatile (M62797) }\end{array}$ & 98,97 \\
\hline DC 8-0-5 (D5) & AY145629 & 290 & $\alpha$-Proteobacteria & $\begin{array}{l}\text { Uncult. clone HP1B39 (AF502216), 'Paracoccus kawasakiensis' } \\
\text { (AB041770) }\end{array}$ & 96,96 \\
\hline DC 8-48-1 (D9) & AY145598 & 1410 & $\alpha$-Proteobacteria & $\begin{array}{l}\text { Uncult. bacterium clone HTH6 (AF418965), bacterial isolate } \\
\text { LD12 (Z99997) }\end{array}$ & 99, 99 \\
\hline DC 8-48-2 (D6) & AY145599 & 537 & Flavo & Rhizosphere soil bacterium clone RSC-II-66 (AJ252690) & 97 \\
\hline DC 8-80-0 (D6) & AY145600 & 510 & $\alpha$-Proteobacteria & $\begin{array}{l}\text { Marine } \alpha \text {-proteobacterium AS-21 (AJ391182), Roseobacter sp. } \\
\text { KT0202a (AF305498) }\end{array}$ & 99,98 \\
\hline DC 8-80-1 (D6) & AY145601 & 1416 & $\gamma$-Proteobacteria & $\begin{array}{l}\text { Uncult. proteobacterium C1_B045 (AF420370), AF094723 } \\
\text { Pseudomonas chlororaphis }\end{array}$ & 92,90 \\
\hline DC 8-80-3 (D5) & AY145602 & 522 & $\alpha$-Proteobacteria & Uncult. $\alpha$-proteobacterium 128-27L (AF473921) & 97 \\
\hline DC 8-80-4 (D5) & AY145603 & 523 & $\alpha$-Proteobacteria & proteobacterium 128-27L (AF473921) & 98 \\
\hline DC 8-80-6 (D5) & AY145604 & 463 & $\beta$-Proteobacteria & $\begin{array}{l}\text { Obligately oligotrophic bacteria PO (AB022337), Methylophilus } \\
\text { methylotrophus (L15475) }\end{array}$ & 98,94 \\
\hline DC 11-0-1 (D7) & AY145605 & 546 & $\beta$-Proteobacteria & $\begin{array}{l}\text { Uncult. freshwater bacterium LD17 (Z99998), Polynucleobacter } \\
\text { necessarius (X93019) }\end{array}$ & 98,96 \\
\hline DC $11-0-2(D 7)$ & AY145606 & 1455 & $\beta$-Proteobacteria & $\begin{array}{l}\text { Uncult. bacterium FukuN33 (AJ289997), uncult. freshwater } \\
\text { bacterium LD17 (Z99998) }\end{array}$ & 97,98 \\
\hline DC 11-0-6 (D6) & AY145607 & 529 & Actinobacteria & $\begin{array}{l}\text { Uncult. actinobacterium WL5-10 (AF497893), Crater Lake clone } \\
\text { CL500-95 (AF316665) }\end{array}$ & 99,97 \\
\hline DC 11-0-7 (D6) & AY145608 & 529 & Actinobacteria & $\begin{array}{l}\text { Uncult. actinobacterium WB5-48 (AF497875), uncult. firmicute } \\
\text { ESR 12 (AF268296) }\end{array}$ & 98,98 \\
\hline DC 11-0-8a (D6) & AY145609 & 546 & $\beta$-Proteobacteria & $\begin{array}{l}\text { Uncult. } \beta \text {-proteobacterium WL11-5 (AF497889), uncult. bacterium } \\
\text { GKS2-122 (AJ290026) }\end{array}$ & 99,98 \\
\hline DC 11-0-8b (D6) & AY145610 & 543 & $\beta$-Proteobacteria & $\begin{array}{l}\text { Uncult. bacterium clone RB7C8 (AF407386), Variovorax sp. } \\
\text { HAB-30 (AB051691) }\end{array}$ & 98,96 \\
\hline DC 11-0-9 (D6) & AY145611 & 548 & $\beta$-Proteobacteria & $\begin{array}{l}\text { Uncult. eubacterium clone FBR-E (AJ319679), Aquaspirillum } \\
\text { delicatum (AF078756) }\end{array}$ & 98,97 \\
\hline DC 11-0-15 (D7) & AY145612 & 529 & Actinobacteria & $\begin{array}{l}\text { Uncult. actinobacterium WB5-42 (AF497876), Crater Lake clone } \\
\text { CL500-95 (AF316665) }\end{array}$ & 99,98 \\
\hline DC 11-0-17 (D7) & AY145613 & 550 & $\beta$-Prot & L15475 Methylophilus methylotrophus & 97 \\
\hline DC 11 & AY145614 & 541 & $\beta$-Proteobacteria & $\begin{array}{l}\text { 3-proteobacterium A0637 (AF236004), Variovorax sp. } \\
\text { HAB-30 (AB051691) }\end{array}$ & 98,97 \\
\hline DC 11-51-0 (D8) & AY145615 & 1457 & $\beta$-Proteobacteria & $\begin{array}{l}\text { Uncult. bacterium clone RB13C10 (AF407413), Rhodoferax } \\
\text { antarcticus (AF084947) }\end{array}$ & 98,96 \\
\hline DC 11-51-1 (D6) & AY145616 & 522 & Actinobacteria & $\begin{array}{l}\text { Uncult. bacterium LES-171 (AJ344205), Actinomyces sp. TM213 } \\
\text { (X92705) }\end{array}$ & 99, 89 \\
\hline DC 11-51-2 (D6) & AY145617 & 522 & $\alpha$-Proteobacteria & $\begin{array}{l}\text { Methylosinus trichosporium (AJ458482), Methylosinus sp. LW8 } \\
\text { (AY007294) }\end{array}$ & 95,94 \\
\hline DC 11-51-4 (D6) & AY145618 & 493 & $\beta$-Proteobacteria & $\begin{array}{l}\text { Uncult. eubacterium WD291 (AJ292624), Acidovorax konjaci } \\
\text { (AF137507) }\end{array}$ & 98,97 \\
\hline DC 11-51-7 (D6) & AY1 & 548 & bacteria & Uncult. clone BIci31 (AJ318113), Methylophilus leisingerii (AF250333) & 3) 98,94 \\
\hline & & & Elav & $\begin{array}{l}\text { Uncult. CFB group bacterium GC1 (AF423372), Microscilla } \\
\text { furvescens (M58792) }\end{array}$ & 7 \\
\hline DC 11-51-10 (D5) & AY145621 & 424 & $\alpha-P r$ & proteobacteriur & 94 \\
\hline & & & $\beta$-Proteobacteria & $\begin{array}{l}\text { Hydrogenophaga pseudoflava (AF078770), uncult. } \beta \text {-proteobacterium } \\
\text { WB8-10B (AF497871) }\end{array}$ & m 95, 94 \\
\hline DC 11-51-14 (D5) & AY145623 & 364 & $\beta$-Proteobacteria & $\begin{array}{l}\beta \text {-proteobacterium JS6666 (AF408397), uncult. } \beta \text {-proteobacterium } \\
\text { WB8-10B (AF497871) }\end{array}$ & 94,94 \\
\hline DC 11-80-1 (D6) & AY145624 & 523 & $\alpha$-Proteobacteria & $\begin{array}{l}\text { Uncult. } \alpha \text {-proteobacterium WM11-37 (AF245632), uncult. } \\
\text { Roseobacter NAC11-3 (AF245632) }\end{array}$ & 99, 99 \\
\hline DC $11-80-2(D 6)$ & AY14 & 1390 & $\alpha$-Prot & Uncult. Roseobacter NAC11-3 (AF245632) & 59 \\
\hline & & & & $\begin{array}{l}\text { Uncul. marine bacterium ZD0202 (AJ400339), Methylophilus } \\
\text { leisingerii (AF250333) }\end{array}$ & \\
\hline DC 11-80-5 (D6) & AY145627 & 479 & $\alpha$-Proteobacteria & $\begin{array}{l}\text { Uncult. } \alpha \text {-proteobacterium WM11-37 (AF245632), uncult. } \\
\text { Roseobacter NAC11-3 (AF245632) }\end{array}$ & 100,100 \\
\hline DC 11-80-6 (D6) & AY145628 & 330 & $\alpha$-Proteobacteria & $\begin{array}{l}\text { Uncult. } \alpha \text {-proteobacterium WM11-37 (AF245632), uncult. } \\
\text { Roseobacter NAC11-3 (AF245632) }\end{array}$ & 100,100 \\
\hline
\end{tabular}



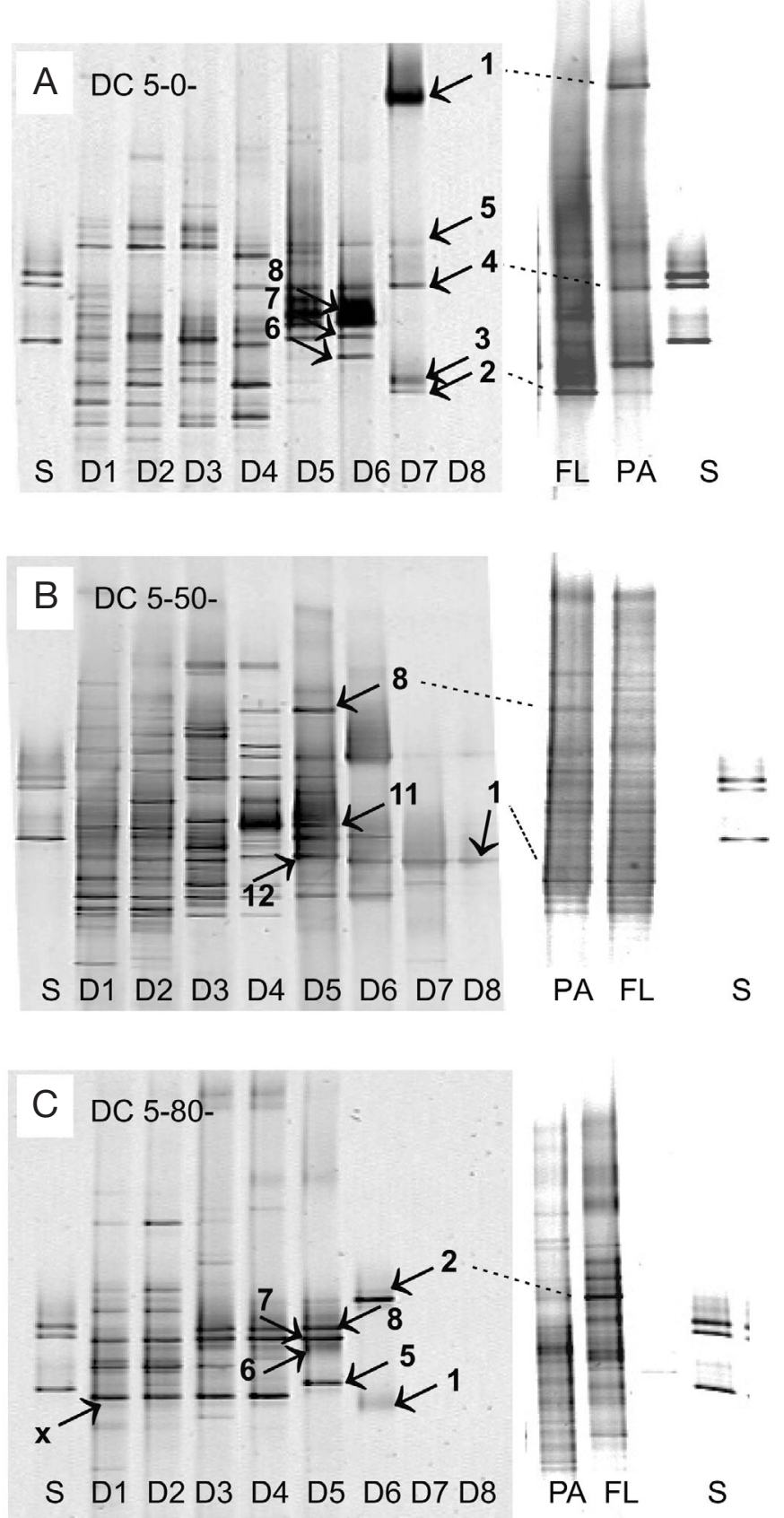

Fig. 2. DGGE analysis of 1 series of increasing dilution steps (D1 $=10^{-1}$ to $\mathrm{D} 8=10^{-8}$ ) of dilution series in (A) limnetic, (B) brackish and (C) marine media in May 1999 in the Weser estuary. Bands on right are original undiluted samples of fractions of free-living (FL) and particle-associated bacteria (PA). S: standard; $\mathbf{X}$ : band extinct in dilution steps $<$ D4. Band numbers refer to sequences in Table 1 \& Fig. 4; dashed lines indicate bands of the undiluted samples that matched those in the dilution cultures (DC)

teria and 2 of $\beta$-Proteobacteria exhibited sequence similarities of $>97 \%$ to bands of the highest dilution steps and/or bands from natural undiluted samples (Table 2). Bands from the natural undiluted samples published in Selje \& Simon (2003) are designated WL-month-band no. (limnetic), WB-month-band no. (brackish) and WM-month-band no. (marine) in Tables and Fig. 4.

Over the entire study period, we ran a negative control at every extraction procedure. Examining this control without any template by PCR, in only 1 case did we detect a contamination which showed $97 \%$ similarity to Microbacterium sp. (AB04207) (Takeuchi \& Hatano 1998). Only 1 DGGE band from our natural samples also affiliated with Microbacterium sp., which, therefore, may represent a contamination in the PCR or extraction reagents.

\section{Bacterial growth on algal extracts and solid/liquid media}

No differences in the composition of bacterial communities growing on the 2 algal substrates were detected by the DGGE patterns (Fig. 3). In contrast, pronounced differences occurred between the bacterial communities growing in the enrichments on solid and liquid media. These differences were substantiated by cluster analysis, which also showed that the evolved communities in the liquid and on the solid media were distinctly different from the sample used as inoculum. Communities in the liquid media clustered even closer than the 2 standards.

\section{Phylogenetic analysis}

\section{$\alpha$-Proteobacteria}

We identified 5 clusters with closely related sequences retrieved from DGGE bands of the original water samples, the higher dilution steps and from isolates (Fig. 4A, Tables 1 \& 3). Of these, 2 clusters contain sequences of limnetic origin, 1 sequences of only brackish origin, 1 sequences of only marine origin, and 1 sequences of both limnetic and brackish origin; 1 limnetic cluster comprising Brevundimonas alba (AF296688) as validated species was defined by Zwart et al. (2002) and Glöckner et al. (2000) as the B. intermedia and alphaII cluster, respectively. Similarities of the sequences within this cluster are at least $98 \%$ (Isolates AP2 and AP3, with 591 bp overlapping) and even a $100 \%$ match for DGGE Band WL5-4 and Dilution Culture Band DC 5-0-4, both retrieved in May (510 bp overlapping). The other limnetic cluster (matching cluster GOBB3-C201 of Zwart et al. 2002) shows a similarity of roughly $95 \%$ to the closest described species Sphingopyxis witflariensis and includes 2 other yet undescribed strains, 1 isolated from the Öre river 
Table 2. Isolates, sequences of bands from highest dilution steps (Dil) of dilution cultures and clone sequences of respective undiluted natural samples from limnetic (WL), brackish (WB) and marine (WM) section of the Weser estuary with sequence similarities of $>97 \%$. Sequences of dilution cultures as in Table 1. Clone sequences of undiluted natural samples were adopted from a previous study (Selje \& Simon 2003). First numbers of clone sequence (DC, WL, WB, WM) indicate month of sampling. Accession no. as in Tables 1, 3 \& 4 and Fig. 4. nd: not detected

\begin{tabular}{|c|c|c|c|c|}
\hline \multirow{2}{*}{ Isolate } & \multicolumn{3}{|c|}{ Dilution culture } & \multirow{2}{*}{$\begin{array}{c}\text { Undiluted } \\
\text { sample }\end{array}$} \\
\hline & Sequence & Dil & Origin & \\
\hline \multicolumn{5}{|c|}{$\alpha$-Proteobacteria } \\
\hline \multirow{7}{*}{ nd } & DC 5-80-2 & D6 & Marine & WM5-49 \\
\hline & DC11-80-1 & D6 & Marine & WM8-15 \\
\hline & DC5-80-3 & D6 & Marine & WM11-37 \\
\hline & DC11-80-2 & D6 & Marine & WB11-28 \\
\hline & DC5-80-4 & D6 & Marine & \\
\hline & DC11-80-5 & D6 & Marine & \\
\hline & DC11-80-6 & D6 & Marine & \\
\hline nd & DC5-50-1 & D8 & Brackish & $\begin{array}{l}\text { WL5-5 } \\
\text { WL5-16 }\end{array}$ \\
\hline nd & DC8-48-1 & D9 & Brackish & WB8-12 \\
\hline AP27 & $\begin{array}{l}\text { DC5-80-8 } \\
\text { DC8-80-0 }\end{array}$ & $\begin{array}{l}\text { D5 } \\
\text { D6 }\end{array}$ & $\begin{array}{l}\text { Marine } \\
\text { Marine }\end{array}$ & nd \\
\hline $\begin{array}{l}\text { AP20 } \\
\text { AP21 }\end{array}$ & DC5-80-6 & D5 & Marine & nd \\
\hline AP1 & DC5-0-4 & D7 & Limnetic & WL5-4 \\
\hline $\begin{array}{l}\text { AP2 } \\
\text { AP3 }\end{array}$ & & & & WL5-15 \\
\hline AP6 & DC8-0-1 & D7 & Limnetic & WL8-22B \\
\hline $\begin{array}{l}\text { AP8 } \\
\text { AP10 }\end{array}$ & & & & WL11-11 \\
\hline $\begin{array}{l}\text { AP10 } \\
\text { AP9-2 }\end{array}$ & DC5-80-5 & D5 & Marine & nd \\
\hline \multicolumn{5}{|c|}{$\beta$-Proteobacteria } \\
\hline BP1 & DC5-0-7 & D6 & Limnetic & nd \\
\hline BP2 & & & & \\
\hline nd & DC11-0-8a & D6 & Limnetic & WL11-5 \\
\hline nd & DC5-50-11 & D5 & Brackish & WL5-9 \\
\hline nd & DC11-51-0 & D8 & Brackish & $\begin{array}{l}\text { WB8-11 } \\
\text { WB5-38B }\end{array}$ \\
\hline \multicolumn{5}{|c|}{ Actinobacteria } \\
\hline nd & DC11-0-7 & D6 & Limnetic & WB5-41 \\
\hline nd & DC11-0-15 & D7 & Limnetic & WL5-10 \\
\hline
\end{tabular}

estuary in northern Sweden (AF321036) (Kisand et al. 2002) and Strain F0813 (AF235997, submitted by Mitsutani et al. directly to GenBank). Similarities of retrieved sequences within Cluster GOBB3-C201 are at least $93 \%$ (Isolate AP9-1 and DGGE Band WL8-22B, with 492 bp overlapping).

The cluster with sequences of limnetic as well as brackish origin belongs to the Rhodobacter-group. Sequences obtained in limnetic natural samples but also in the highest dilution step $\left(10^{-8}\right)$ in the brackish medium (DC8-0-5, DC5-0-6, DC5-50-1) in May show more than $99 \%$ similarity and are most closely (96\%) related to $R$. capsulatus (D16428). The sequences of brackish origin of the DGGE bands from the natural sample and the highest dilution step $\left(10^{-9}\right)$ in August affiliate with the freshwater SAR11 cluster (Bahr et al.
1996), named LD12 by Zwart et al. (2002), and are similar to the alphaV cluster of Glöckner et al. (2000).

In the marine section, we repeatedly found almost identical clones ( $\geq 99 \%$ ) affiliating with the Roseobacter clade and with uncultured Roseobacter NAC11-3 (Gonzalez et al. 2000) as the next known sequence. These clones were detected in May and November 1999 in all parallels of the highest dilution step in which growth occurred (Table 1, Fig. 4A). Sequences of this closely related cluster, which was designated Roseobacter-clade affiliated cluster (RCA, Selje et al. 2004), were also obtained from the most intense band in the DGGE profiles from the undiluted samples of all sampling dates (Selje \& Simon 2003).

In addition, quite a few sequences of the Roseobacter clade were retrieved from DGGE bands of various natural samples (Selje \& Simon 2003) and higher dilution steps (Table 1). However, only 2 isolates affiliated with this clade (Table 3): AP27 was isolated from the $10^{-6}$ dilution culture in May, which exhibited a prominent DGGE band with an identical sequence (523 bp overlapping). In August, an almost identical sequence was detected in the $10^{-6}$ dilution culture also (99\% identity,

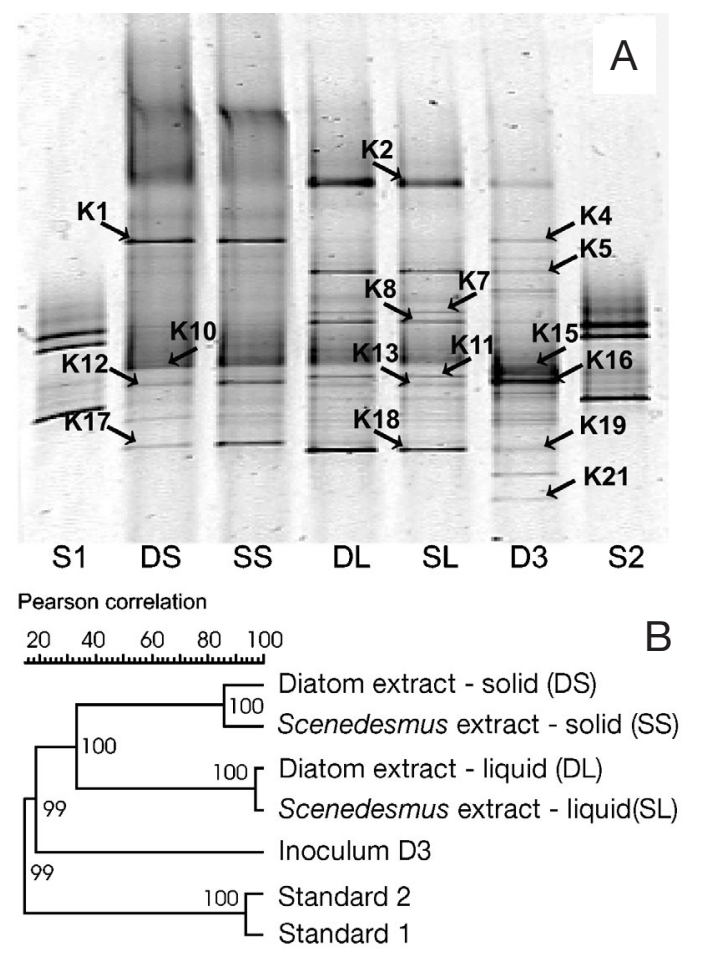

Fig. 3. (A) Inverted DGGE pattern and (B) cluster analysis of bacterial communities from Dilution Step $10^{-3}$ (D3) of a dilution culture from May 1999 and from enrichment cultures on solid, S (DS, SS), and liquid, L (DL, SL), media inoculated with D3. Enrichments were extracts from diatoms (D) or Scenedesmus sp. (S). S1, S2: standard 1, standard 2. Band designations as in Table 4. Cluster analysis was based on Pearson correlation and UPGMA. Numbers in dendrogram indicate calculated cophenetic correlations 
Fig. 4. Phylogenetic trees of Weser estuary sequences and clones (boldface) of dilution cultures (DC) from May, August and November 1999, isolates, clones from enrichment cultures and bands obtained from DGGE analyses of undiluted natural samples from limnetic (WL), brackish (WB) and marine (WM) sections. Clone sequences of undiluted natural samples were adopted from a previous study (Selje \& Simon 2003). (L), (B), and (M) after DC identifications indicate limnetic, brackish and marine origin of the dilution culture samples, respectively.

(A) $\alpha$-Proteobacteria with selected clusters; (B) $\beta$-Proteobacteria with selected clusters; (C) $\gamma$-Proteobacteria; (D) Flavobacteria/ Sphingobacteria group of Bacteroidetes phylum; (E) Gram-positive bacteria including Actinobacteria and Bacillales. Backbone tree based on maximum likelihood analysis was constructed with almost complete (>1300 nucleotides) sequences. Alignment positions at which $<50 \%$ of the corresponding sequences had the same residues were excluded. Sequences shorter than 1300 nucleotides were added with maximum parsimony using the same filter. For accession numbers of sequences obtained in this study see

Tables 1, 3 \& 4

(1)
$\left[\begin{array}{ccc}-\mathrm{DC} 5-80-2(\mathrm{M}) \\ -\mathrm{DC} 11-80-1) \\ \mathrm{DC}-80-3(\mathrm{M})\end{array}\right.$

$-D C 5-80-3(M)$
$D C 11-80-2(M)$

Uncult. Roseobacter

(AF 245632)

Uncult. Roseobacter sp. (AF353235)

WB119 (AF497859),

5-80-4 (M), DC11-80-5 (M), DC11-80-6 (M)

Octadecabacter antarcticus (U14583)
Leisingera methylohalidivorans (AY005463)

Roseobacter gallaeci

[ Isolate AP26

Marine alpha Proteobacterium AS-21 (AJ391182)

Roseobacter sp. KT0202a (AF305498)

Isolate AP27 DC 5-80-8 (M)

DC 8-80-0 (M)

CVSP bacterium CV919-312 (AF114484)
DC 8-80-3 (M)

DC 8-80-4 (M)

DC 5-80-7 (M)

Sulfitobacter pontiacus (Y13155)

Uncultobacter mediterraneus (Y17387)

-14 (AF497863), WM11-36 (AF497864)

Isolate AP15
APeria atlantica (D88526)

Roseobacter

clade
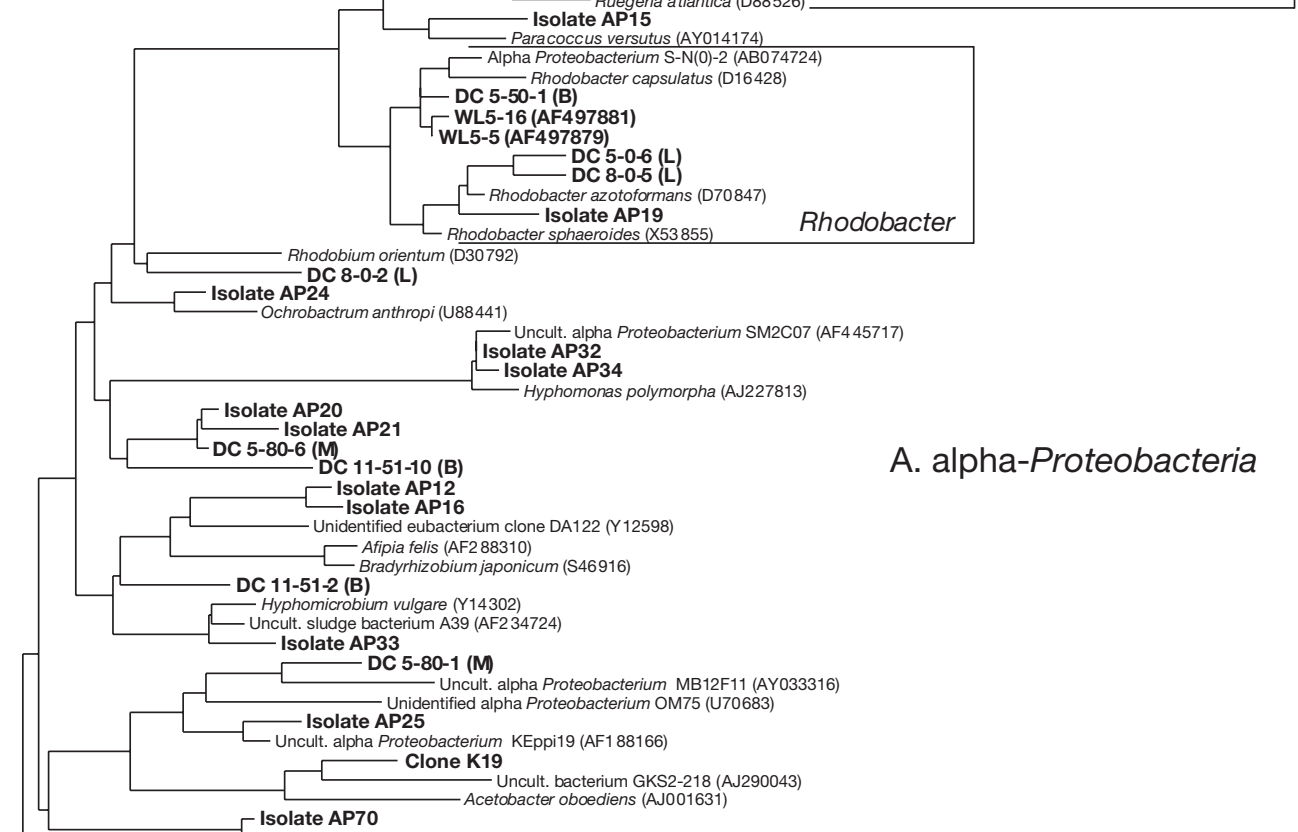

A. alpha-Proteobacteria

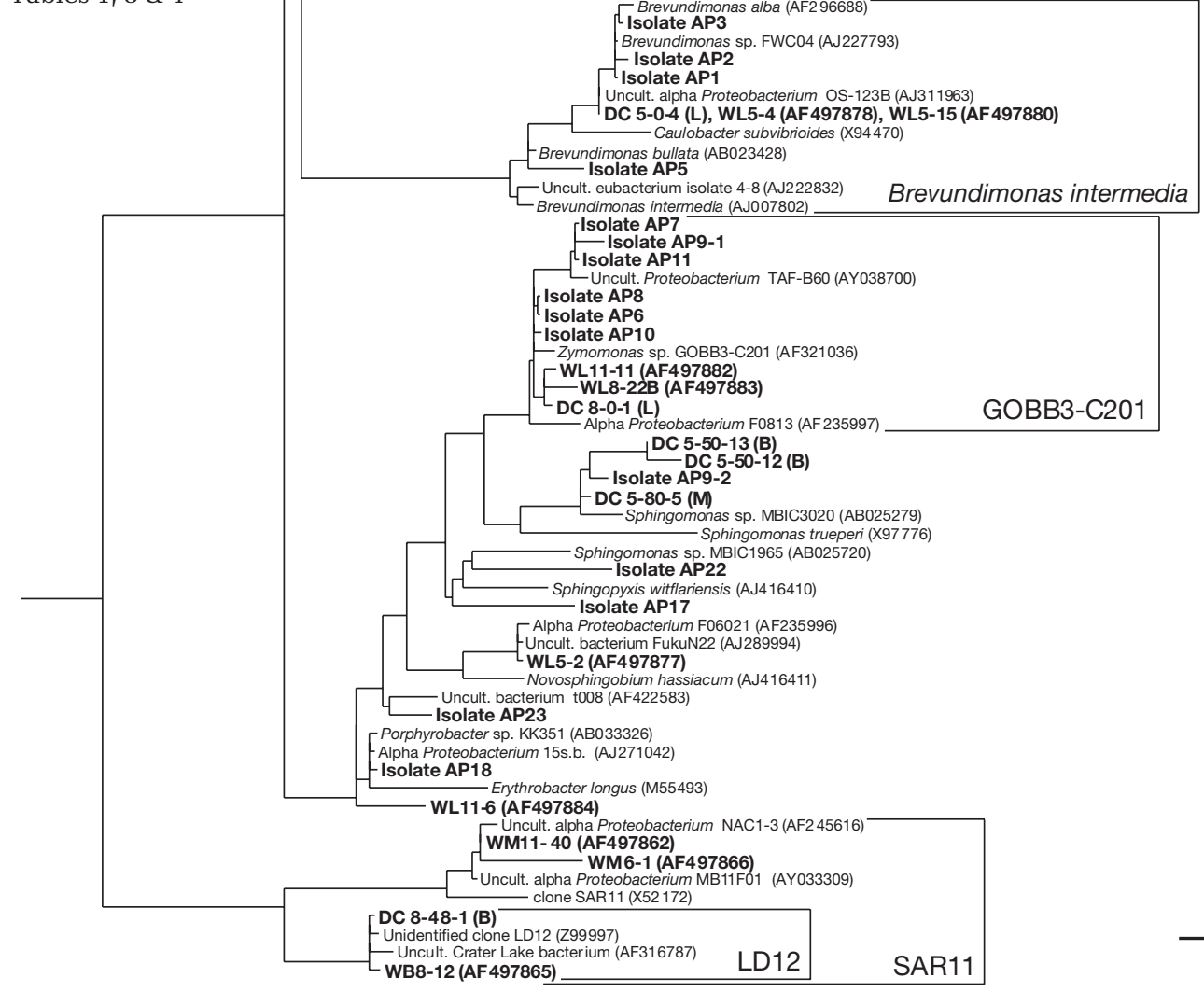

0.1 
B. beta-Proteobacteria (Fig. 4 continued)

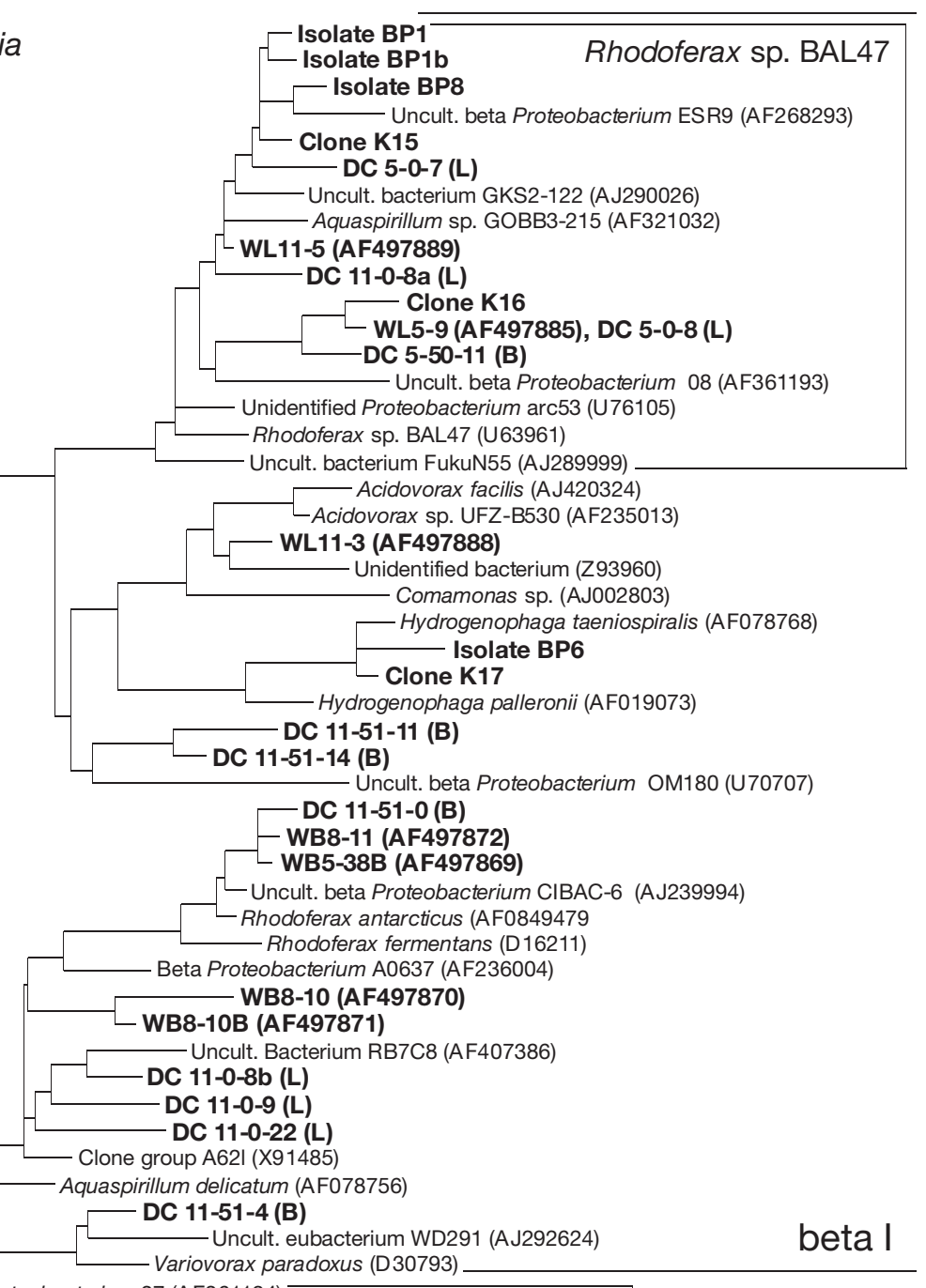

-Variovorax paradoxus (D30793)

\section{Polynucleobacter} necessarius

Polynucleobacter necessarius (X93019)

Uncult freshwater bacterium LD17 (Z99998)

- DC 11-0-2 (L)

DC 11-0-1 (L)

Uncult. beta Proteobacterium clone CR-FL23 (AF141405)

Clone K13

- Ultramicrobacterium str. MY14 (AB008503)

Herbaspirillum seropedicae (Y10146)

Methylophilus leisingerii (AF250333)

Methylophilus methylotrophus (L15475)

DC 11-0-17 (L)

DC 11-80-3 (M)

Unidentified beta Proteobacterium OM44 (U70704)

- Obligately oligotrophic bacterium (AB022337)

DC 8-80-6 (M)

- Uncult. freshwater bacterium LD28 (Z99999)

- Unidentified beta Proteobacterium ACK-C30 (U85120)

Uncult. beta Proteobacterium clone CR-FL21 (Af141403) Isolate BP5

Beta Proteobacterium clone PRD01a011B (AF289159)

DC 11-51-7 (B)

Uncult. beta Proteobacterium Blqi38 (A J318162)

WL8-23 (AF497886) 


\section{C. gamma-Proteobacteria}

(Fig. 4 continued)

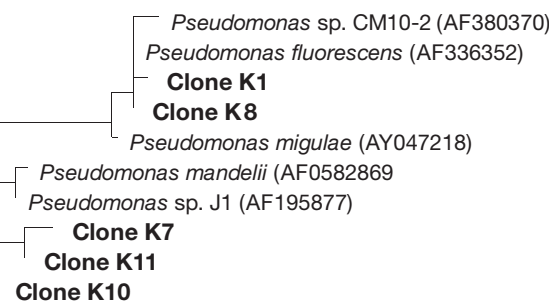

Pseudomonas sp. CM10-2 (AF380370) Pseudomonas fluorescens (AF336352)

Clone K1

\section{Clone K8}

Pseudomonas migulae (AY047218)

- Pseudomonas mandelii (AF0582869

Pseudomonas sp. J1 (AF195877)

- Clone K7

Clone K11

Clone K10

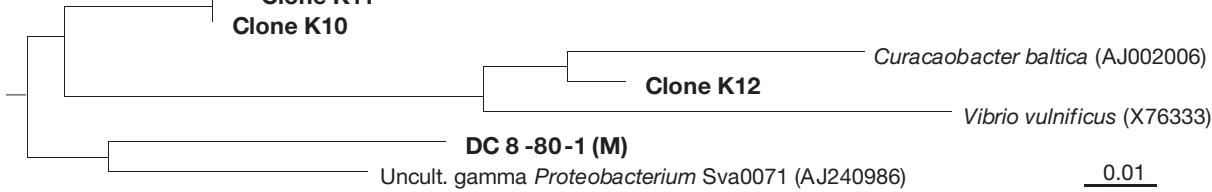

\section{Flavobacteria/Sphingobacteria}

(Fig. 4 continued)

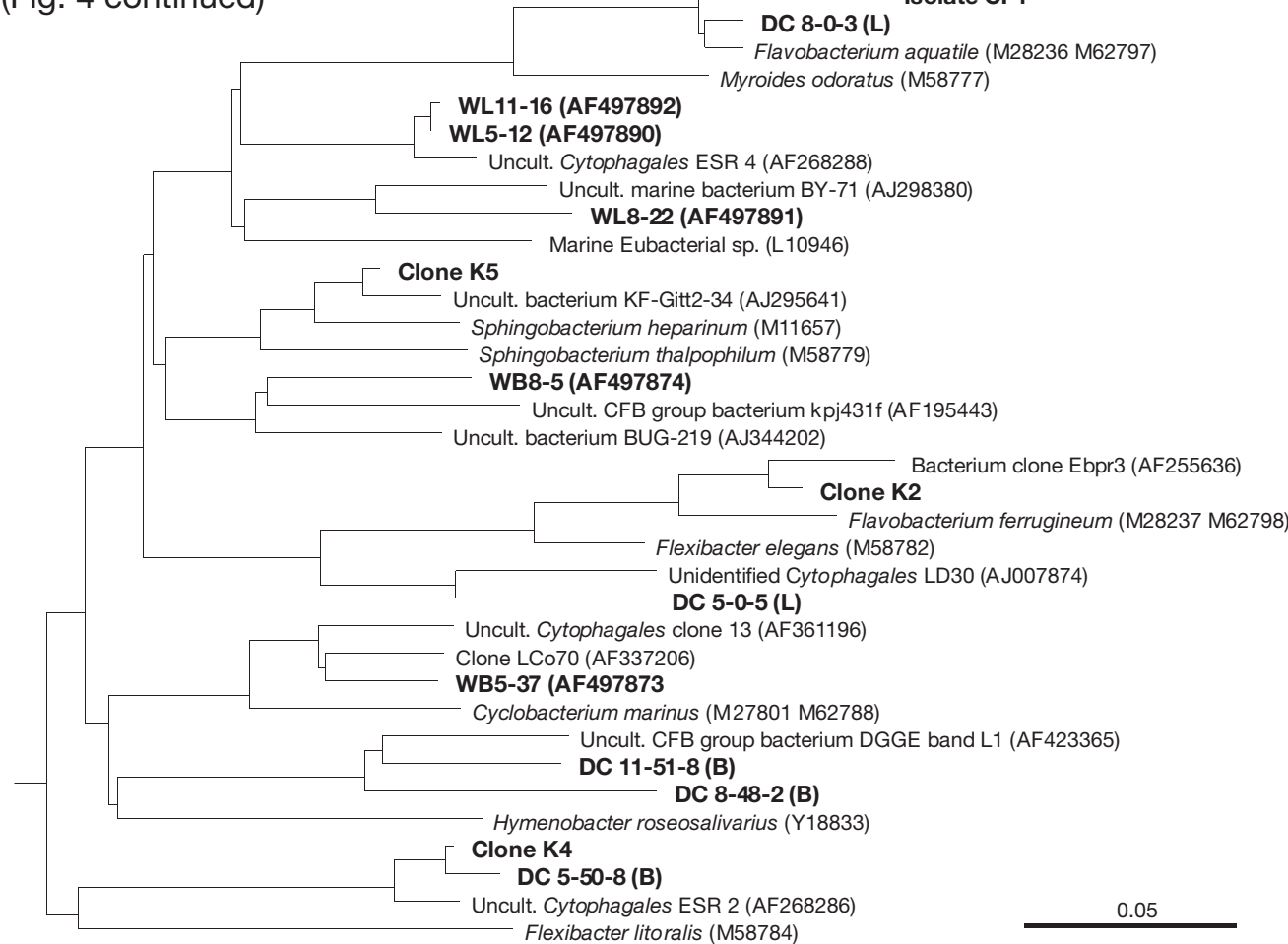

509 bp overlapping). These sequences were related most closely to strains isolated from the Adriatic Sea (AJ391182, Fajon et al. direct submission to GenBank) and the North Sea (AF305498, Eilers et al. 2001). Our isolate AP26 is also related most closely to a strain isolated from the Adriatic Sea (AJ391174, Fajon et al. direct submission to GenBank).

\section{$\beta$-Proteobacteria}

Most of the retrieved sequences of $\beta$-Proteobacteria fell into the betaI cluster of Glöckner et al. (2000). The sequences which occurred most frequently affiliated with a distinct subcluster, the Rhodoferax sp. BAL47 cluster described by Zwart et al. (2002; Fig. 4B, Tables 1 \& 3). They were most closely related (96 to $97 \%$ similarity) to Aquaspirillum delicatum (AF078756) as next validly described species. Of the 2 almost full-length 16S rRNA gene sequences retrieved from this group, 1 was obtained from the inoculum of the brackish section in November 1999 and affiliated with the betaI cluster as well, but with Rhodoferax antarcticus as the closest described isolated strain. The corresponding DGGE band was also detected in the brackish samples in May and August (Table 2). The other almost full-length sequence was retrieved from a limnetic culture in November 1999 and affiliated with 


\section{E. Gram-positive Bacteria} (Fig. 4 continued)

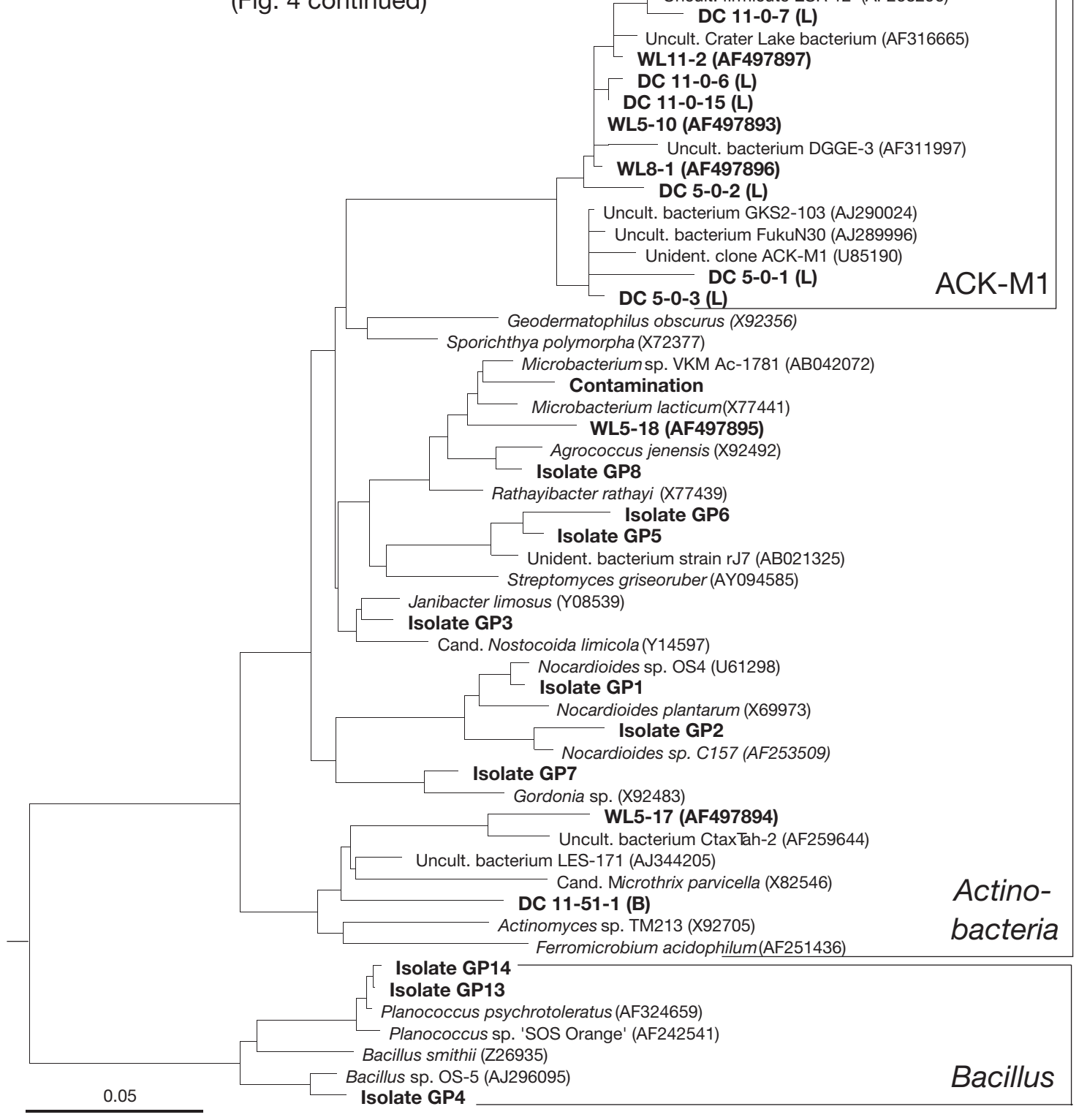

the Polynucleobacter necessarius cluster (Zwart et al. 2002). A related sequence was also detected in the limnetic section of the estuary in August.

Even though most of the sequences affiliated with $\beta$ Proteobacteria were retrieved from the limnetic or brackish section and dilution cultures, 2 DGGE bands were retrieved from marine dilution cultures (DC8-086 , DC11-80-3), indicating that some $\beta$-Proteobacteria are able to grow at salinities up to 28 . The next related sequences were a clone retrieved from the coastal picoplankton of Cape Hatteras, USA (U70704, Rappé et al. 1997) and an obligately oligotrophic strain isolated by Katanozaka \& Yoshinaga (AB022337, GenBank, direct submission), respectively. One limnetic sequence (DC11-0-17) clustered closely together with these marine sequences. As this cluster includes Methylophilus methylophilus, we propose calling it 'Methylophilus cluster'.

\section{$\gamma$-Proteobacteria}

Only 1 sequence of the higher dilution cultures (DC8-80-1) and no isolate and clone of natural samples affiliated with $\gamma$-Proteobacteria. However, we obtained quite a few sequences affiliating with this phylogenetic group and closely related to Pseudomonas sp. and Curaçaobacter baltica in the enrichments on solid 
and liquid media (Table 4). Interestingly, there were no related sequences found among the bands excised from the community in the inoculum used (Lane 6 [D3] in Fig. 3).

\section{Flavobacteria/Sphingobacteria}

Bacteria of the Flavobacteria/Sphingobacteria group were detected less frequently than those of $\alpha$ - and $\beta$ Proteobacteria. Only 5 of the 44 intense and sequenced bands from the DGGE banding patterns of natural undiluted samples affiliated with this group, and only 5 of 56 sequences from the dilution cultures and 1 of the isolates. The latter showed only low (96\%) and decreasing sequence similarity to the next known sequence (uncultured bacterium AF087055), a band detected in a dilution culture of August, and to the next validly described species, Flavobacterium aquatile (M28236 M62797, $94 \%$, Tables 1 \& 3).

\section{Gram-positive bacteria}

Within the Gram-positive bacteria, isolates were obtained from Actinobacteria as well as from the Bacillus group. However, none of the latter sequences matched any sequence of DGGE bands from the natural undiluted samples or from the dilution cultures. All except 1 of these sequences affiliated within Actinobacteria with Cluster ACK-M1 (Zwart et al. 2002), identical to Cluster hgcI (Glöckner et al. 2000). Tree construction (Fig. 4E) and calculated distance matrix values showed Sporotricha polymorpha (X72377) to be the most closely related species.

\section{Other phylogenetic groups}

The growth experiment yielded 2 additional bands from bacterial groups undetected hitherto in this study. Clone K21 from the evolved bacterial community in the $10^{-3}$ dilution step affiliated with the Nitrospira group, and Clone K18 from the liquid medium fell into the $\delta$-Proteobacteria, with Melittangium boletus (AJ233908) being the closest relative according to BLAST results.

\section{DISCUSSION}

The aim of this study was to detect abundant members of the natural bacterial community in the Weser estuary using a PCR-dependent and a culture dependent approach, i.e. dilution cultures, and to ob- tain representative isolates. In a previous study in this estuary we showed by using the DGGE technique that along the salinity gradient distinct bacterial communities exist which partly overlap and undergo seasonal changes (Selje \& Simon 2003). The results of the present study show that sequences of the few or single bands in the high dilution steps and of isolates matched those of prominent DGGE bands of natural undiluted samples (Table 2). Hence, our dilution culture approach with natural sample water as substrate and diluting out less abundant but faster-growing bacteria, provided prominent bacteria with favorable growth conditions.

It has been argued that cloning protocols and different efficiencies in primer annealing may introduce selective amplification and discrimination against specific bacterial clusters, thus biasing the validity of clone libraries and DGGE-based analyses with respect to the composition of ambient bacterial communities (Rainey et al. 1994, Cottrell \& Kirchman 2000, Bano \& Hollibaugh 2002, O'Sullivan et al. 2002). Therefore, it was important to validate the PCR-based approach with 1 PCR-independent and 1 culture dependent approach. The fact that we obtained 16S rRNA gene sequences in high dilution steps, matching sequences of prominent DGGE bands of the natural samples, indicated that the combination of these 2 approaches was successful. Further, obtaining almost complete 16S rRNA gene sequences of a single prominent bacterium in the highest dilution steps, matching the sequences of prominent DGGE bands of the natural undiluted samples, made it possible to extend the limited sequence information of the 16S rRNA gene fragments generated by the DGGE approach. Similarly, Bano \& Hollibaugh (2002) extended the limited sequence information of cloned DGGE bands in a study in the Arctic Ocean. They compared DGGE banding patterns of $16 \mathrm{~S}$ rRNA gene fragments amplified directly with those of almost full-length clone libraries from the same locations and thus were able to attribute almost full-length sequences to prominent DGGE bands.

Currently, studies examining the composition of ambient natural bacterioplankton communities are usually not based on culture-dependent methods, but on the application of PCR-based approaches. To our knowledge, this is the first study which has examined the composition of bacterioplankton communities by the dilution culture technique and subsequent DGGE analysis, and has found corresponding results in quite a few cases. The great majority of bacteria growing in the highest dilution steps affiliated with phylogenetic clusters of which phylotypes or isolates were found in other studies from limnetic, brackish and marine environments (Giovannoni \& Rappé 2000, Glöckner et al. 2000, Bruns et al. 2002, 2003a, Zwart et al. 2002, Hahn 
Table 3. Isolates from the Weser estuary with accession numbers (Acc. no.), dilution step (Dil), medium and estuarine section (Medium), month in which they were obtained, numbers of base pairs (bp) sequenced, closest relatives according to BLAST search, and sequence similarity

\begin{tabular}{|c|c|c|c|c|c|}
\hline Isolate & (Acc. no.) & Dil, Medium, Date & bp & Closest relative (Acc. no.) & nilarity $(\%)$ \\
\hline \multicolumn{6}{|c|}{ Gram-positive bacteria } \\
\hline GP-1 & (AY145529) & $10^{4}$ marine, August 99 & 1102 & Nocardioides OS4 (U61298) & 98 \\
\hline GP-2 & (AY145530) & $10^{3}$, brackish, May 99 & 916 & Nocardioides sp. C157 (AF253509) & 97 \\
\hline GP-3 & (AY145531) & $10^{6}$, brackish, May 00 & 643 & Janibacter limosus (Y08539) & 96 \\
\hline GP-4 & (AY145532) & $10^{8}$, brackish, May 00 & 1143 & Bacillus sp. OS-5 (AJ296095) & 98 \\
\hline GP-5 & (AY145533) & $10^{7}$, brackish, August 99 & 1361 & $\begin{array}{l}\text { Unidentified bacterium strain rJ7 (AB021325), Sporichthya } \\
\text { polymorpha (AB025317) }\end{array}$ & 97,94 \\
\hline GP-6 & $(\mathrm{AY} 145534)$ & $10^{6}$, brackish, August 99 & 1246 & $\begin{array}{l}\text { Unident. bacterium strain rJ7 (AB021325), Sporichthya } \\
\text { brevicatena (AB006164) }\end{array}$ & 96,92 \\
\hline GP-7 & (AY145535) & $10^{4}$, limnetic, August 99 & 694 & Gordona sp. (X92483) & $\begin{array}{l}90,92 \\
99\end{array}$ \\
\hline GP-8 & (AY145536) & $10^{7^{\prime}}$, limnetic, May 99 & 1074 & Agrococcus jenensis (X92492) & 98 \\
\hline GP-13 & (AY145537) & $10^{5}$, marine, August 99 & 1385 & 'Planococcus psychrotoleratus' (AF324659) & 99 \\
\hline GP-14 & (AY145538) & $10^{5}$, marine, August 99 & 1422 & 'Planococcus psychrotoleratus' (AF324659) & 99 \\
\hline \multicolumn{6}{|c|}{ Flavobacteria/Sphingobacteria } \\
\hline $\mathrm{CF}-1$ & (AY145539) & $10^{7}$, limnetic, May 99 & 1412 & $\begin{array}{l}\text { Uncultured bacterium BS3 (AF087055), Flavobacterium } \\
\text { aquatile (M62797 M28236) }\end{array}$ & 96,94 \\
\hline \multicolumn{6}{|c|}{$\alpha$-Proteobacteria } \\
\hline AP-1 & $(\mathrm{AY} 145540)$ & $10^{6}$, brackish, May 99 & 995 & Brevundimonas sp. strain FWC04 (AJ227793) & 99 \\
\hline AP-2 & (AY145541) & $10^{6}$, limnetic, May 99 & 1237 & Brevundimonas sp. strain FWC04 (AJ227793) & 99 \\
\hline AP-3 & (AY145542) & $10^{7^{\prime}}$, limnetic, August 99 & 694 & Brevundimonas sp. strain FWC04 (AJ227793) & 99 \\
\hline AP-5 & (AY145543) & $10^{6}$, limnetic, August 99 & 1216 & Brevundimonas bullata strain: MBIC2745 (AB023428) & 97 \\
\hline AP-6 & $(\mathrm{AY} 145544)$ & $10^{7^{\prime}}$, limnetic, August 99 & 1369 & $\alpha$-proteobacterium F0813 (AF235997), Sphingopyxis witflariensis & \multirow{2}{*}{$\begin{array}{l}97,95 \\
96,94\end{array}$} \\
\hline AP-7 & $(\mathrm{AY} 145545)$ & $10^{7}$, limnetic, May 99 & 1330 & $\alpha$-proteobacterium F0813 (AF235997), Sphingopyxis witflariensis & \\
\hline AP-8 & $(\mathrm{AY} 145546)$ & $10^{7}$, limnetic, August 99 & 1357 & a-proteobacterium F0813 (AF235997), Sphingopyxis witflariensis & 96,94 \\
\hline AP-9-1 & $(\mathrm{AY} 145547)$ & $10^{7}$, limnetic, May 99 & 1266 & $\begin{array}{l}\text { (AJ416410) } \\
\alpha \text {-proteobacterium F0813 (AF235997), Sphingopyxis witflariensis }\end{array}$ & \multirow{2}{*}{$\begin{array}{c}9 f, 95 \\
96,95 \\
98\end{array}$} \\
\hline AP_9-2 & (AY145548) & $10^{7}$ limnetic Mav 99 & 1038 & $\begin{array}{l}\text { (AJ416410) } \\
\text { Sphingomonas sp MBIC3020 (AF025352) }\end{array}$ & \\
\hline AP-10 & (AY145549) & $10^{7}$, limnetic, August 99 & 700 & Uncult. clone TAF-B60 (AY038700), Zymomonas sp. GOBB3-C201 & \multirow{2}{*}{98,98} \\
\hline AP-11 & (AY145550) & $10^{7}$, limnetic, May 99 & 695 & Uncult. clone TAF-B60 (AY038700), $\alpha$-proteobacterium F0813 & \\
\hline AP-12 & $(\mathrm{AY} 145551)$ & $10^{5}$, limnetic, August 99 & 1332 & $\begin{array}{l}\text { (AF235997) } \\
\text { Unidentified clone DA122 (Y12598), Afipia felis (AF288310) }\end{array}$ & $\begin{array}{l}99,96 \\
93,92\end{array}$ \\
\hline AP-15 & (AY145552) & $10^{9}$, brackish, August 99 & 677 & Paracoccus versutus (AY014174) & 96 \\
\hline AP-16 & (AY145553) & $10^{5}$, limnisch, August 99 & 1265 & Unidentified clone DA122 (Y12598), Afipia felis (AF288310) & 94,92 \\
\hline AP-17 & $(\mathrm{AY} 145554)$ & $10^{6}$, limnetic, August 99 & 670 & Blastobacter sp. SMCC B0477 (U20772), Erythromonas ursincola & 94,94 \\
\hline AP-18 & $(\mathrm{AY} 145555)$ & $10^{5}$, limnetic, August 99 & 1028 & $\alpha$-proteobacterium strain 15s.b. (AJ271042), Porphyrobacter sp. & 9999 \\
\hline AP-19 & (AY145556) & $10^{5}$, marine, May 99 & 672 & Rhodobacter azotoformans (D70847) & 95 \\
\hline AP-20 & (AY145557) & $10^{5^{\prime}}$, marine, May 99 & 900 & Ochrobactrum anthropi (AF501340) & 92 \\
\hline AP-21 & (AY145558) & $10^{5}$, marine, May 99 & 1381 & Uncultured soil bacterium clone 60-2 (AF423281), Mesorhizobium & \multirow{2}{*}{92,92} \\
\hline AP-22 & (AY145559) & $10^{5}$, marine, May 99 & 1275 & Sphingomonas sp. D16 (AF025352), Sphingomonas sp. MBIC1965 & \\
\hline AP-23 & $(\mathrm{AY} 145560)$ & $10^{4}$, limnetic, August 99 & 814 & Uncult. clone t008 (AF422583), Agrobacterium sanguineum & \multirow{2}{*}{$\begin{array}{l}98,86 \\
98\end{array}$} \\
\hline AP-24 & (AY145561) & $10^{6}$, brackish, August 99 & 681 & Ochrobactrum anthropi (U88441) & \\
\hline AP- 25 & (AY145562) & $10^{6}$, marine, August 99 & 1002 & Uncult. $\alpha$-proteobacterium KEppib19 (AF188166), Azospirillum sp. & 98.89 \\
\hline AP-26 & (AY145563) & $10^{6}$, brackish, August 99 & 1340 & Marine $\alpha$-proteobacterium AS-11(AJ391174), Roseobacter & 99,95 \\
\hline AP-27 & $(\mathrm{AY} 145564)$ & $10^{6}$, marine, May 99 & 1347 & Marine $\alpha$-proteobacterium AS-21 (AJ391182), Roseobacter sp. & \multirow{2}{*}{99,99} \\
\hline AP-32 & (AY145565) & $10^{6}$, limnetic, August 99 & 988 & Uncult. $\alpha$-proteobacterium SM2C07 (AF445717), Hyphomonas & \\
\hline AP-33 & (AY145566) & $10^{6}$, limnetic, August 99 & 679 & $\begin{array}{l}\text { jannaschiana (AF082789) } \\
\text { Hyphomicrobium vulgare (Y14302), Hyphomicrobium W1-1B }\end{array}$ & \multirow{2}{*}{97,98} \\
\hline AP-34 & $(\mathrm{AY} 145567)$ & $10^{6}$, limnetic, August 99 & 661 & $\begin{array}{l}\text { (U59505) } \\
\text { Hyphomonas rosenbergii (AF082795) }\end{array}$ & \\
\hline AP-70 & (AY145568) & $10^{3}$, brackish, May 99 & 968 & Methylobacterium fujisawaense (AJ250801) & 99 \\
\hline $\begin{array}{l}\beta \text {-Protec } \\
\mathrm{BP}-1\end{array}$ & $\begin{array}{l}\text { obacteria } \\
\text { (AY145569) }\end{array}$ & $10^{7}$, limnetic, May 99 & 1454 & $\begin{array}{l}\text { Uncult. bacterium GKS2-122 (AJ290026), Aquaspirillum delicatum } \\
\text { (AF078756) }\end{array}$ & 97,97 \\
\hline $\mathrm{BP}-1 \mathrm{~b}$ & $(\mathrm{AY} 145570)$ & $10^{7}$, limnetic, May 99 & 1454 & Uncult. bacterium GKS2-122 (AJ290026), Aquaspirillum delicatum & 97 \\
\hline BP-5 & (AY145571) & $10^{4}$, limnetic, August 99 & 1466 & Uncult. $\beta$-proteobacterium PRD01a011B (AF289159), & \multirow[b]{2}{*}{$\begin{array}{l}95,91 \\
98\end{array}$} \\
\hline BP-6 & $(\mathrm{A}$ & $10^{3}$, brackish, May 99 & 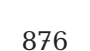 & piralis (AF078768) & \\
\hline BP-8 & (AY145573) & $10^{7^{\prime}}$, limnetic, May 99 & 728 & $\begin{array}{l}\text { Uncult. } \beta \text {-proteobacterium } 08 \text { (AF361201), Aquaspirillum sp. } \\
\text { GOBB3-215 (AF321032) }\end{array}$ & 98,98 \\
\hline
\end{tabular}


Table 4. Phylogenetic affiliation and closest related sequences of clones obtained from dilution step 10 $0^{-3}$ (D3) from the dilution culture of May 1999 and from enrichment cultures inoculated with D3 samples. Enrichments were on media with additions of extracts from diatoms or Scenedesmus sp. Given are clone identifications, accession numbers (Acc. no.), type of medium or dilution step (DS: solid medium with diatom extract; SL: liquid medium with Scenedesmus sp. extract), number of base pair (bp), closest related sequences and sequence similarity

\begin{tabular}{|llllll|}
\hline Clone & Acc. no. & Medium & bp & Closest relative (Acc. no.) & Similarity (\%) \\
\hline K1 & AY146656 & DS & 546 & Pseudomonas sp. CM10-2 (AF380370) & 98 \\
K2 & AY146657 & SL & 541 & Uncultured bacterium clone Ebpr3 (AF255636) & 98 \\
K4 & AY146658 & D3 & 542 & Uncult. Cytophagales ESR 2 (AF268286) & 95 \\
K5 & AY146659 & D3 & 541 & Uncult. bacterium KF-Gitt2-34 (AJ295641) & 98 \\
K7 & AY146660 & SL & 546 & Pseudomonas migulae (AY047218) & 99 \\
K8 & AY146661 & SL & 546 & Pseudomonas fluorescens (AF336352) & 98 \\
K10 & AY146662 & DS & 546 & Pseudomonas sp. J1 (AF195877) & 98 \\
K11 & AY146663 & SL & 546 & Pseudomonas sp. J1 (AF195877) & 96 \\
K12 & AY146664 & DS & 546 & Curacaobacter baltica OS 140 (AJ002006) & 99 \\
K13 & AY146665 & SL & 546 & Herbaspirillum sp. BA17 (AF364861) & 99 \\
K15 & AY146666 & D3 & 546 & Uncult. $\beta$-proteobacterium WL11-5 (AF47889) & 99 \\
K16 & AY146667 & D3 & 546 & Uncult. $\beta$-proteobacterium WL5-9 (AF47885) & 98 \\
K17 & AY146668 & DS & 548 & Hydrogenophaga taeniospiralis (AF078768) \\
K18 & AY146669 & SL & 456 & Melittangium boletus, strain Me b8 (AJ233908) & 90 \\
K19 & AY146670 & D3 & 522 & Uncult. bacterium GKS2-218 (AJ290043) & 98 \\
K21 & AY146671 & D3 & 536 & Nitrospira sp. strain RC14 (Y14637) & \\
\hline
\end{tabular}

2003). The sequences of $\alpha$-Proteobacteria affiliated with 3 well known limnetic and 2 marine clusters, those of $\beta$-Proteobacteria with 3 clusters and Actinobacteria sequences with 1 cluster. Hence, our study on the basis of this culture dependent approach yielded a similar composition of the bacterioplankton communities as studies based on PCR approaches and thus provides a valuable support for the latter. It further demonstrates that these bacteria are in fact active components of the ambient bacterioplankton community and can be enriched by the dilution culture approach.

In addition to the enrichment of highly abundant bacteria in the highest dilution steps, we obtained a cultivation efficiency of more than $1.5 \%$ and up to $66 \%$ (Fig. 1). Such cultivation efficiencies are significantly higher than on solid media and are in line with previous reports applying the same approach without any or with low nutrient addition, or with addition of only signal molecules such as cAMP and acetyl homoserine lactones (Button et al. 1993, Bussmann et al. 2001, Jaspers et al. 2001, Bruns et al. 2002, 2003a). This approach also enabled us to isolate bacteria from dilution steps of $10^{-6}$ to $10^{-9}$, thus close to dilution steps at which growth was no longer detected (Table 3). More than $30 \%$ of the isolates had a sequence similarity of $<96 \%$ to described species. Usually, limnetic and brackish samples yielded isolates at higher dilution steps than marine samples. According to the rationale of this approach, these isolates are presumed to be abundant in the original water sample and thus potentially prominent members of the ambient bacterial community. Supporting evidence is provided by the fact that in several cases prominent DGGE bands of these isolates occurred in the original water sample (Table 2). Also, other studies successfully applied the MPN approach to isolate abundant planktonic bacteria, such as members of the SAR11 clade (Rappé et al. 2002) and a marine actinobacterium, constituting $1 \%$ of bacterial numbers in the German Wadden Sea (Bruns et al. 2003b).

In quite a few cases we were not able to isolate on solid media bacteria that were enriched in the highest dilution steps and occurred as prominent DGGE bands in the original water sample, e.g. $\alpha$-Proteobacteria of the marine RCA cluster, the freshwater SAR11 (LD12) and ACK-M1 clusters. The marine RCA cluster, with a sequence similarity of more than $98 \%$, is globally distributed in temperate to polar oceans but absent from subtropical and tropical regions (Selje et al. 2004). Bacteria of this cluster not only occurred as prominent DGGE bands, but also constituted 3 to $10 \%$ of the bacterioplankton community in the Weser estuary and the German Bight, as shown by a quantitative PCR approach with cluster-specific primers and FISH with cluster-specific probes (Selje et al. 2004). Possible reasons for the detection of these bacteria in the higher dilution steps but their failure to as pure culture on solid media include a continuous dependence on metabolites from other (micro)organisms which were missing from the growth medium, a limited capacity of divisions on the basis of storage products, or the inability to grow on solid media. Bussmann et al. (2001) found an increased cultivability with the MPN technique on low $(0.06 \%)$ compared to higher $(0.6 \%)$ substrate concentrations, and the bacteria from the former 
enrichments were not able to form colonies on solid agar. Schut et al. (1993) found indications that organic matter additions to dilution cultures inhibited bacterial growth. Also in other studies, the MPN approach failed to isolate abundant planktonic bacteria (Button et al. 1993, Bruns et al. 2002, 2003a). On the other hand, other isolation approaches using solid media with low nutrient additions and $0.2 \mu \mathrm{m}$ filtered samples and subsequent substrate acclimation recently yielded new isolates of planktonic bacteria clusters for which previously only uncultured phylotypes had been known (Kisand et al. 2002, Hahn 2003, Hahn et al. 2003).

$\alpha$-Proteobacteria were most prominent among the sequenced bands of the higher dilution steps and the isolates: $83 \%$ of the bands from the marine samples ( $\mathrm{n}=37), 27 \%$ of the limnetic and brackish samples $(\mathrm{n}=$ 18) and $67 \%$ of the isolates in both the marine and limnetic/brackish samples $(\mathrm{n}=44)$ belonged to this phylogenetic subclass. $\beta$-Proteobacteria constituted $41 \%$ of the DGGE bands in the limnetic/brackish samples, but only $11 \%$ of the isolates. Hence, our dilution culture approach with long incubation times and low nutrient concentrations seemed to favor the growth of $\alpha$-Proteobacteria in particular in limnetic/brackish samples. Eilers et al. (2001) also found an increased proportion of marine $\alpha$-Proteobacteria among colonyforming bacteria at longer incubation times and at reduced ammonium and phosphate concentrations compared to artificial seawater medium.

It is important to point out that this dilution-toextinction approach with no extra nutrient addition did not cause a preferential growth of $\gamma$-Proteobacteria, as we obtained only 1 band in the higher dilution steps and no isolate of this phylogenetic subclass. A preferential growth of $\gamma$-Proteobacteria in limnetic as well as in marine samples growing on nutrient-enriched solid media has often been observed (Wagner et al. 1993, Eilers et al. 2000a, Fuchs et al. 2000). In the experiment comparing the growth of bacteria on enriched solid and in liquid media, however, quite a few sequences affiliating with $\gamma$-Proteobacteria were obtained, in particular with Pseudomonas sp. and with Curaçaobacter baltica (Table 4). Interestingly, there were no related sequences among the bands excised from the DGGE patterns of the inoculum, indicating that bacteria developed which did not appear to be abundant under in situ conditions. Hence, by adding only low amounts of mixed 'natural' substrates and inoculating with a sample diluted only to $10^{-3}$, we already induced a 'gamma-shift', not only on the solid, but also in the liquid medium.

In line with the results of the dilution cultures, we reported in a previous paper that no $\gamma$-Proteobacteria were among the sequenced bands from the DGGE profiles of the original samples from different seasons and sections of the Weser estuary (Selje \& Simon 2003). This observation may have been due to primer discrimination or because we happened to miss these bands for excision and subsequent sequence analysis, suggesting that $\gamma$-Proteobacteria were not among the most intense bands on our DGGE profiles. The culturedependent approach may also have discriminated against this phylogenetic group, or members of this group could, in fact, be of little significance in the Weser estuary. In the German Bight of the North Sea $\gamma$-Proteobacteria have been detected by FISH, and constitute 10 to $>20 \%$ of the DAPI cell counts; they thus appear to be a prominent component of the bacterioplankton in this area (Eilers et al. 2001).

In the limnetic and brackish section, we detected $\beta$-Proteobacteria in the highest dilution steps which matched phylotypes in the natural bacterial community by DGGE (Table 2). We also obtained isolates from the subcluster which comprised most of the phylotypes, Rhodoferax sp. BAL47. From this subcluster, with which quite a few phylotypes in limnetic systems affiliate (Glöckner et al. 2000, Zwart et al. 2002), 2 other isolates in addition to ours are known (Pinhassi et al. 1997, Kisand et al. 2002). In line with many other studies, these findings indicate that $\beta$-Proteobacteria are important components of the actively growing limnetic and brackish bacterioplankton (e.g. Pernthaler et al. 1998, Glöckner et al. 1999, Zwisler et al. 2003).

We also obtained growth of $\beta$-Proteobacteria in high dilution steps in the marine medium, supporting our observation in a previous paper that this phylogenetic group constituted $6 \%$ of the DAPI cell counts of the particle-associated bacteria in the marine section detected by FISH (Selje \& Simon 2003). So far, marine $\beta$-Proteobacteria are known only from a distinct cluster within the ammonium-oxidizers and from a cluster closely related to Methylophilus sp. (Rappé et al. 1997, Phillips et al. 1999). These bacterial groups exhibit a rather limited substrate spectrum and, therefore, are presumably not of general significance in marine carbon cycling. It has been shown that ammoniumoxidizers affiliating with $\beta$-Proteobacteria account for $<1 \%$ of total bacteria in limnetic and brackish systems (Ward et al. 1997). Hence, they are under the detection limit of DGGE. We assume that for the same reason we did not detect $\beta$-Proteobacteria by DGGE in the natural samples from the marine section (Selje \& Simon 2003).

In the highest dilution steps, only $9 \%$ of the clones affiliated with the Flavobacteria/Sphingobacteria group and we obtained only 1 isolate. These results appear surprising because in other studies in marine as well as in limnetic systems based on FISH and clone libraries bacteria of this phylogenetic lineage were found to be more abundant (Böckelmann et al. 2000, Cottrell \& Kirchman 2000, Kirchman 2002, O'Sullivan et al. 2002, 
Kirchman et al. 2003). In the Weser estuary, Flavobacteria/Sphingobacteria comprised 14 to $28 \%$ of DAPI cell counts in the particle-associated bacterial community, as determined by FISH (Selje \& Simon 2003). A similar discrepancy was found by Böckelmann et al. (2000), who reported that Flavobacteria/Sphingobacteria constituted up to $36 \%$ of the DAPI cell counts on aggregates in the River Elbe, but only $1 \%$ among 40 isolates.

There are several possible explanations for the low numbers and proportions of Flavobacteria/Sphingobacteria, in particular in relation to $\alpha$-Proteobacteria. (1) A selective PCR bias, perhaps due to mismatches of Bacteria-specific primers for the 16S rRNA gene (Cottrell \& Kirchman 2000, Suzuki et al. 2001, Kirchman et al. 2003). The application of specific primers for this bacterial group yielded substantially higher numbers of phylotypes compared to general bacterial primers (O'Sullivan et al. 2002, Kirchman et al. 2003, Rink et al. 2003). Further, the copy number of $16 \mathrm{~S}$ rRNA genes may affect PCR-amplification. $\alpha$-Proteobacteria harbor 1 to 4 SSU rRNA genes, Actinobacteria 1 to $6, \gamma$-Proteobacteria 4 to 10 and Rhodothermus marinus (as the only example of the Flavobacteria/Sphingobacteria group) harbor 1 (Fogel et al. 1999). (2) Flavobacterial Sphingobacteria grew slowly because of unfavorable growth conditions in the dilution cultures. This argument, however, holds true only if a PCR-bias occurred simultaneously, because this group was underrepresented by both methods. Such a dual bias seems unlikely, since we obtained good agreements with $\alpha$ and $\beta$-Proteobacteria and Actinobacteria. (3) The richness of the Flavobacteria/Sphingobacteria group was rather high, and single phylotypes, therefore, are below the detection limit of $1 \%$ in the DGGE analysis (Muyzer et al. 1993, Murray et al. 1996) and dilutedout in the highest dilution steps. In fact, in the German Wadden Sea, a high diversity within the Flavobacteria/Sphingobacteria group was found by applying DGGE with primers specific for this group (Rink et al. 2003). The effect of this phenomenon, however, is difficult to distinguish and separate from a possible PCR bias. (4) The Flavobacteria/Sphingobacteria group in the free-living bacterial community is much less abundant than in the particle-associated fraction, which accounted for 11 and $50 \%$ of total cell counts in the limnetic and brackish sections (Selje \& Simon 2003). Thus, members of this group account for 3 and $8 \%$ of total bacteria plus an unknown fraction in the free-living community. Assuming this unknown fraction to be around $10 \%$, the proportion of this group would be in the range of 11 to $14 \%$ of total bacteria, and thus in the range of numbers of phylotypes detected by DGGE, and only slightly higher than detection efficiencies by the dilution culture approach.
Considering all arguments raised, we assume that we slightly underestimated the number of phylotypes in the DGGE and the dilution culture approach due to primer bias and that the solid media discriminated against the Flavobacteria/Sphingobacteria group in the same way as shown for the other phylogenetic groups.

\section{CONCLUSIONS}

The combination of a PCR-dependent and the dilution culture-dependent approach enabled us to show that in the Weser estuary prominent bacteria were enriched in and eventually isolated from the highest dilution steps of the dilution cultures, thus emphasizing their abundance in situ. The 2 approaches yielded complementary results and extend previous findings with molecular techniques, i.e. the bacterioplankton community in various aquatic systems is dominated by subclusters of distinct phylogenetic groups. Information on these groups is based predominantly on $16 \mathrm{~S}$ rRNA gene sequences of phylotypes. Using the dilution approach with ambient substrates at a low concentration, bacteria of these groups could be enriched and subsequently isolated. For a more comprehensive understanding of the biogeochemical role of these bacteria, the revelation of their physiology in general and under in situ conditions is most important. A critical step was the transfer to solid media, and therefore several strains, defied isolation. dilution cultures appear to be suitable for the growth of important members of the bacterioplankton, and hence more emphasis needs be laid on applying this promising technique.

Acknowledgements. We are most grateful to the crew of the RV 'Bakensand', in particular to R. Schütte from the NLWK, for their excellent cooperation with the field work. We thank R. Brinkmeyer and 2 anonymous referees for stylistic suggestions and constructive criticism on an earlier version of this manuscript. This work was supported by a grant from the Deutsche Forschungsgemeinschaft (Si 360/10-1).

\section{LITERATURE CITED}

Bahr M, Hobbie JE, Sogin ML (1996) Bacterial diversity in an Arctic lake: a freshwater SAR11 cluster. Aquat Microb Ecol 11:271-277

Bano N, Hollibaugh JT (2002) Phylogenetic composition of bacterioplankton assemblages from the Arctic Ocean. Appl Environ Microbiol 68:505-518

Böckelmann U, Manz W, Neu TR, Szewzyk U (2000) Characterization of the microbial community of lotic organic aggregates ('river snow') in the Elbe River of Germany by cultivation and molecular methods. FEMS Microbiol Ecol 33:157-170

Bruns A, Cypionka H, Overmann J (2002) Cyclic AMP and acyl homoserine lactones increase the cultivation efficiency of heterotrophic bacteria from the central Baltic 
Sea. Appl Environ Microbiol 68:3978-3987

Bruns A, Nübel U, Cypionka H, Overmann J (2003a) Effects of signal compounds and incubation conditions on the culturability of freshwater bacterioplankton. Appl Environ Microbiol 69:1980-1989

Bruns A, Philipp H, Cypionka, Brinkhoff T (2003b) Aeromicobium marinum sp. nov., an abundant pelagic bacterium isolated from the German Wadden Sea. Int J Syst Evol Microbiol 53: 1917-1923

Bussmann I, Philipp B, Schink B (2001) Factors influencing the cultivability of lake water bacteria. J Microbiol Methods $47: 41-50$

Button DK, Schut F, Quang P, Martin R, Robertson BR (1993) Viability and isolation of marine bacteria by dilution culture; theory, procedures, and initial results. Appl Environ Microbiol 59:881-891

Cole JJ, Findlay S, Pace ML (1988) Bacterial production in fresh and saltwater ecosystems: a cross-system overview. Mar Ecol Prog Ser 43:1-10

Cottrell MT, Kirchman DL (2000) Community composition of marine bacterioplankton determined by $16 \mathrm{~S}$ rRNA gene clone libraries and fluorescence in situ hybridization. Appl Environ Microbiol 66:5116-5122

Eguchi M, Ishida Y (1990) Oligotrophic properties of heterotrophic bacteria and in situ heterotrophic activity in pelagic seawaters. FEMS Microbiol Ecol 73:23-30

Eilers H, Pernthaler J, Amann R (2000a) Succession of pelagic marine bacteria during enrichment: a close look at cultivation-induced shifts. Appl Environ Microbiol 66:4634-4640

Eilers H, Pernthaler J, Glöckner FO, Amann R (2000b) Culturability and in situ abundance of pelagic bacteria from the North Sea. Appl Environ Microbiol 66:3044-3051

Eilers H, Pernthaler J, Peplies J, Glöckner FO, Gerdts G, Amann R (2001) Isolation of novel pelagic bacteria from the German Bight and their seasonal contributions to surface picoplankton. Appl Environ Microbiol 67:5134-5142

Farrelly V, Rainey F, Stackebrandt E (1995) Effect of genome size and rrn gene copy number on PCR amplification of 16S rRNA genes from a mixture of bacterial species. Appl Environ Microbiol 61:2798-2801

Fogel GB, Collins CR, Li J, Brunk CF (1999) Prokaryotic genome size and SSU rDNA copy number: estimation of microbial relative abundance from a mixed population. Microb Ecol 38:93-113

Fuchs BM, Zubkov MV, Sahm K, Burkill PH, Amann R (2000) Changes in community composition during dilution cultures of marine bacterioplankton as assessed by flow cytometric and molecular biological techniques. Environ Microbiol 2:191-201

Fuhrman JA, McCallum K, Davis AA (1993) Phylogenetic diversity of subsurface marine microbial communities from the Atlantic and Pacific Ocean. Appl Environ Microbiol 59:1294-1302

Giovannoni S, Rappé M (2000) Evolution, diversity, and molecular ecology of marine prokaryotes. In: Kirchman D (ed) Microbial ecology of the oceans. Wiley-Liss, New York, p 47-84

Giovannoni SJ, Britschgi TB, Moyer CL, Field KG (1990) Genetic diversity in Sargasso Sea bacterioplankton. Nature 345:60-63

Glöckner FO, Fuchs BM, Amann R (1999) Bacterioplankton compositions of lakes and oceans: a first comparison based on fluorescence in situ hybridization. Appl Environ Microbiol 65:3721-3726

Glöckner FO, Zaichikov E, Belkova N, Denissova L, Pernthaler J, Pernthaler A, Amann R (2000) Comparative 16S rRNA analysis of lake bacterioplankton reveals globally distributed phylogenetic clusters including an abundant group of Actinobacteria. Appl Environ Microbiol 66: 5053-5065

Gonzalez JM, Moran MA (1997) Numerical dominance of a group of marine bacteria in the $\alpha$-subclass of the class Proteobacteria in coastal seawater. Appl Environ Microbiol 63:4237-4242

Gonzalez JM, Simo R, Massana R, Covert J, Casamayor E, Pedros-Alio C, Moran MA (2000) Bacterial community structure associated with a dimethylsulfonioproponiateproducing North Atlantic algal bloom. Appl Environ Microbiol 66:4237-4246

Hahn MW (2003) Isolation of strains belonging to the cosmopolitan Polynucleobacter necessarius cluster from freshwater habitats located in three climatic zones. Appl Environ Microbiol 69:5248-5254

Hahn MW, Lünsdorf $H$, Wu Q, Schauer M, Höfle MG, Boenigk J, Stadler P (2003) Isolation of ultramicrobacteria classified as Actinobacteria from five freshwater habitats in Europe and Asia. Appl Environ Microbiol 69:1442-1451

Haller CM, Rolleke S, Vybiral D, Witte A, Velimirov B (2000) Investigation of 0.2 micron filterable bacteria from the Western Mediterranean Sea using a molecular approach: dominance of potential starvation forms. FEMS Microbiol Ecol 31:153-161

Ishii K, Fukui M (2001) Optimization of annealing temperature to reduce bias caused by a primer mismatch in multitemplate PCR. Appl Environ Microbiol 67:3753-3755

Jannasch H (1958) Studies of planktonic bacteria by means of a direct membrane filter method. J Gen Microbiol 18: $609-620$

Jaspers E, Nauhaus K, Cypionka H, Overmann J (2001) Multitude and temporal variability of ecological niches as indicated by the diversity of cultivated bacterioplankton. FEMS Microbiol Ecol 36:153-164

Kirchman DL (2002) The ecology of Cytophaga-Flavobacteria in aquatic environments. FEMS Microbiol Ecol 1317: 91-100

Kirchman DL, Yu L, Cottrell MT (2003) Diversity and abundance of uncultured Cytophaga-like bacteria in the Delaware estuary. Appl Environ Microbiol 69:6587-6596

Kisand V, Cuadros R, Wikner J (2002) Phylogeny of culturable estuarine bacteria catabolizing riverine organic matter in the northern Baltic Sea. Appl Environ Microbiol 68: 379-388

Klee AJ (1993) A computer program for the determination of most probable number and its confidence limits. J Microbiol Methods 18:91-98

Kuznetsov SI (1975) The microflora of lakes and its geochemical activity. University of Texas Press, Austin, TX

Ludwig W, Strunk O, Westram R, Richter L and 28 others (2004) ARB: a software environment for sequence data. Nucleic Acids Res 32:1363-1371

Murray AE, Hollibaugh JT, Orrego C (1996) Phylogenetic compositions of bacterioplankton from two California estuaries compared by denaturing gradient gel electrophoresis of $16 \mathrm{~S}$ rDNA fragments. Appl Environ Microbiol 62:2676-2680

Muyzer G, De Waal EC, Uitterlinden AG (1993) Profiling of complex microbial populations by denaturing gradient gel electrophoresis analysis of polymerase chain reactionamplified genes coding for 16S rRNA. Appl Environ Microbiol 59:695-700

Muyzer G, Teske A, Wirsen CO, Jannasch HW (1995) Phylogenetic relationships of Thiomicrospira species and their identification in deep-sea hydrothermal vent samples by denaturing gradient gel electrophoresis of 16S rRNA fragments. Arch Microbiol 164:165-172 
Muyzer G, Brinkhoff T, Nübel U, Santegoeds C, Schäfer H, Wawer C (1998) Denaturing gradient gel electrophoresis (DGGE) in microbial ecology. In: Akkermans ADL, van Elsas JD, Bruijn FJ (eds) Molecular microbial ecology manual. Kluwer Academic, Dordrecht, p 1-23

Nübel U, Garcia-Pichel F, Muyzer G (1997) PCR primers to amplify 16S rRNA genes from cyanobacteria. Appl Environ Microbiol 63:3327-3332

O'Sullivan LA, Weightman AJ, Fry JC (2002) New degenerate Cytophaga-Flexibacter-Bacteroides-specific $16 \mathrm{~S}$ ribosomal DNA-targeted oligonucleotide probes reveal high bacterial diversity in River Taff epilithon. Appl Environ Microbiol 68:201-210

Pernthaler J, Glöckner FO, Unterholzner S, Alfreider A, Psenner R, Amann R (1998) Seasonal community and population dynamics of pelagic Bacteria and Archaea in a high mountain lake. Appl Environ Microbiol 64:4299-4306

Phillips CJ, Smith Z, Embley TM, Prosser JI (1999) Phylogenetic differences between particle-associated and planktonic ammonia-oxidizing bacteria of the beta subdivision of the class proteobacteria in the northwestern Mediterranean Sea. Appl Environ Microbiol 65:779-786

Pinhassi J, Zweifel UL, Hagström A (1997) Dominant marine bacterioplankton species found among colony-forming bacteria. Appl Environ Microbiol 63:3359-3366

Polz MF, Cavanaugh CM (1998) Bias in template-to-product ratios in multitemplate PCR. Appl Environ Microbiol 64:3724-3730

Rainey FA, Ward N, Sly LI, Stackebrandt E (1994) Dependence on the taxon composition of clone libraries for PCR amplified, naturally occurring $16 \mathrm{~S}$ rDNA, on the primer pair and the cloning system used. Experientia 50:796-797

Rappé MS, Kemp PF, Giovannoni SJ (1997) Phylogenetic diversity of marine coastal picoplankton 16S rRNA genes cloned from the continental shelf off Cape Hatteras, North Carolina. Limnol Oceanogr 42:811-826

Rappé MS, Connon SA, Vergin KL, Giovannoni SJ (2002) Cultivation of the ubiquitous SAR11 marine bacterioplankton clade. Nature 418:630-633

Razumov AS (1932) The direct method of calculation of bacteria in water: comparison with the Koch method. Mikrobiologiya 1:131-146 (in Russian)

Reysenbach A, Giver L, Wickham G, Pace N (1992) Differential amplification of rRNA genes by polymerase chain reaction. Appl Environ Microbiol 58:3417-3418

Rink B, Lunau M, Seeberger S, Stevens H, Brinkhoff $T$, Grossart HP, Simon M (2003) Diversity patterns of aggregate-associated and free-living bacterial communities in the German Wadden Sea. Ber ForschZent Terramare 12: 96-98

Schmidt TM, DeLong EF, Pace NR (1991) Analysis of a marine picoplankton community by $16 \mathrm{~S}$ rRNA gene cloning and sequencing. J Bacteriol 173:4371-4378

Editorial responsibility: Karel Šimek,

České Budějovice, Czech Republic
Schut F, de Vries EJ, Gottschal JC, Robertson BR, Harder W, Prins RA, Button DK (1993) Isolation of typical marine bacteria by dilution culture: growth, maintenance, and characteristics of isolates under laboratory conditions. Appl Environ Microbiol 59:2150-2160

Selje N, Simon M (2003) Composition and dynamics of particle-associated and free-living bacterial communities in the Weser estuary, Germany. Aquat Microb Ecol 30:221-237

Selje N, Simon M, Brinkhoff T (2004) A newly discovered Roseobacter cluster in temperate and polar oceans. Nature 427:445-448

Staley JT, Konopka A (1985) Measurement of in situ activities of nonphotosynthetic microorganisms in aquatic and terrestrial habitats. Annu Rev Microbiol 39:321-346

Suzuki MT, Giovannoni SJ (1996) Bias caused by template annealing in the amplification of mixtures of 16S rRNA genes by PCR. Appl Environ Microbiol 62:625-630

Suzuki MT, Béjà O, Taylor LT, DeLong EF (2001) Phylogenetic analysis of ribosomal RNA operons from uncultivated coastal marine bacterioplankton. Environ Microbiol 3:323-342

Takeuchi M, Hatano K (1998) Union of the genera Microbacterium Orla-Jensen and Aureobacterium Collins et al. in a redefined genus Microbacterium. Int J Syst Bacteriol 48:739-747

Urbach E, Vergin KL, Young L, Morse A, Larson GL, Giovannoni SJ (2001) Unusual bacterioplankton community structure in ultra-oligotrophic Crater Lake. Limnol Oceanogr 46:557-572

van Es FB, Meyer-Reil LA (1982) Biomass and metabolic activity of heterotrophic marine bacteria. Adv Microb Evol $6: 111-170$

Wagner M, Amann R, Lemmer H, Schleifer KH (1993) Probing activated sludge with oligonucleotides specific for proteobacteria: inadequacy of culture dependent methods for describing microbial community structure. Appl Environ Microbiol 59:1520-1525

Ward B, Voytec M, Witzel KP (1997) Phylogenetic diversity of natural populations of ammonia oxidizers investigated by specific PCR amplification. Microb Ecol 33:87-96

Zwart G, Hiorns WD, Methe BA, van Agterveld MP, Huismans R, Nold SC, Zehr JP, Laanbroek HJ (1998) Nearly identical 16S rRNA sequences recovered from lakes in North America and Europe indicate the existence of clades of globally distributed freshwater bacteria system. Syst Appl Microbiol 21:546-556

Zwart G, Crump BC, Kamst-van Agterveld MP, Hagen F, Han SK (2002) Typical freshwater bacteria: an analysis of available 16S rRNA gene sequences from plankton of lakes and rivers. Aquat Microb Ecol 28:141-155

Zwisler W, Selje N, Simon M (2003) Seasonal patterns of the bacterioplankton community composition in a large mesotrophic lake. Aquat Microb Ecol 31:211-225

Submitted: September 10, 2003; Accepted: December 20, 2004 Proofs received from author(s): April 7, 2005 